\title{
PCA-Based Spatially Adaptive Denoising of CFA Images for Single-Sensor Digital Cameras
}

\author{
Lei Zhang, Member, IEEE, Rastislav Lukac, Member, IEEE, Xiaolin Wu, Senior Member, IEEE, and \\ David Zhang, Fellow, IEEE
}

\begin{abstract}
Single-sensor digital color cameras use a process called color demosaicking to produce full color images from the data captured by a color filter array (CFA). The quality of demosaicked images is degraded due to the sensor noise introduced during the image acquisition process. The conventional solution to combating CFA sensor noise is demosaicking first, followed by a separate denoising processing. This strategy will generate many noise-caused color artifacts in the demosaicking process, which are hard to remove in the denoising process. Few denoising schemes that work directly on the CFA images have been presented because of the difficulties arisen from the red, green and blue interlaced mosaic pattern, yet a well designed "denoising first and demosaicking later" scheme can have advantages such as less noise-caused color artifacts and cost-effective implementation. This paper presents a principle component analysis (PCA) based spatially-adaptive denoising algorithm, which works directly on the CFA data using a supporting window to analyze the local image statistics. By exploiting the spatial and spectral correlations existed in the CFA image, the proposed method can effectively suppress noise while preserving color edges and details. Experiments using both simulated and real CFA images indicate that the proposed scheme outperforms many existing approaches, including those sophisticated demosaicking and denoising schemes, in terms of both objective measurement and visual evaluation.
\end{abstract}

Index Terms-Adaptive denoising, Bayer pattern, color filter array (CFA), demosaicking, principle component analysis (PCA).

\section{INTRODUCTION}

$\mathbf{M}$ OST existing digital color cameras use a single sensor with a color filter array (CFA) [11] to capture visual scenes in color. Since each sensor cell can record only one color value, the other two missing color components at each position need to be interpolated from the available CFA sensor readings to reconstruct the full-color image. The color interpolation process is usually called color demosaicking (CDM). Many CDM algorithms [2]-[11], [14]-[17] proposed in the past

Manuscript received May 06, 2008; revised November 06, 2008. Current version published March 13, 2009. This work was supported by the CERG of Hong Kong under Grant B-Q06F and the National Science Foundation Council of China under Grant 60634030. The associate editor coordinating the review of this manuscript and approving it for publication was Prof. Stanley J. Reeves.

L. Zhang and D. Zhang are with the Department of Computing, The Hong Kong Polytechnic University, Hong Kong (e-mail: cslzhang@ comp.polyu.edu. hk).

R. Lukac is with Epson Edge, Epson Canada, Ltd., Toronto, ON M1W $3 Z 5$ Canada.

$\mathrm{X}$. Wu is with the Department of Electrical and Computer Engineering, McMaster University, Hamilton, ON L8S 41K Canada.

Color versions of one or more of the figures in this paper are available online at http://ieeexplore.ieee.org.

Digital Object Identifier 10.1109/TIP.2008.2011384 are based on the unrealistic assumption of noise-free CFA data. The presence of noise in CFA data not only deteriorates the visual quality of captured images, but also often causes serious demosaicking artifacts which can be extremely difficult to remove using a subsequent denoising process. Note that many advanced denoising algorithms [19]-[26], which are designed for monochromatic (or full color) images, are not directly applicable to CFA images due to the underlying mosaic structure of CFAs. To overcome the problem, we will propose a principle component analysis (PCA)-based denoising scheme which directly operates on the CFA domain of captured images.

Though most existing CDM techniques [3]-[10], [14]-[17] assume noise-free CFA data, this assumption does not hold well in practice. For almost all kinds of color imaging devices, ranging from the low-cost and/or resource-constrained ones such as wireless camera phones to the high-end ones such as digital cinema cameras, image corruptive noise is inherent and can be severe; thus, the restoration of color images from noisy CFA data is a challenging problem. To suppress the effect of noise on the demosaicked image, three strategies are possible: denoising after demosaicking; denoising before demosaicking; and joint demosaicking-denoising.

An intuitive and convenient strategy to remove noise is to denoise the demosaicked images. Algorithms developed for gray-scale imaging, for example [19]-[26], can be applied to each channel of the demosaicked color image separately whereas some color image filtering techniques [12], [13] process color pixels as vectors. The problem of this strategy is that noisy sensor readings are roots of many color artifacts in demosaicked images and those artifacts are difficult to remove by denoising the demosaicked full-color data. In general the CFA readings corresponding to different color components have different noise statistics. The CDM process blends the noise contributions across channels, thus producing compound noise that is difficult to characterize. This makes the design of denoising algorithms for single-sensor color imaging very difficult.

Recently, some schemes that perform demosaicking and denoising jointly have been proposed [27]-[34]. In [33], Trussell and Hartig presented a mathematical model for color demosaicking using minimum mean square error (MMSE) estimator. The additive white noise is considered in the modeling. Ramanath and Snyder [34] proposed a bilateral filter based demosaicking method. Since bilateral filtering exploits the similarity in both spatial and intensity spaces, this scheme can handle light noise corrupted in the CFA image. Hirakawa and Parks [27] developed a joint demosaicking-denoising algorithm by using the total least square (TLS) technique where both demosaicking 
and denoising are treated as an estimation problem with the estimates being produced from the available neighboring pixels. The filter used for joint demosaicking-denoising is determined adaptively using the TLS technique under some constraints of the CFA pattern. In [28] and [29], Hirakawa et al. proposed two wavelet based schemes that can perform CDM simultaneously with denoising. The joint demosaicking-denoising scheme developed by Zhang et al. [30] first performs demosaicking-denoising on the green channel. The restored green channel is then used to estimate the noise statistics in order to restore the red and blue channels. In implementing the algorithm, Zhang et $a l$. estimated the red-green and blue-green color difference images rather than directly recovering the missing color samples by using a linear model of the color difference signals. Inspired by the directional linear minimum mean square-error estimation (DLMMSE) based CDM scheme in [9], Paliy et al. [31], [32] proposed an effective nonlinear and spatially adaptive filter by using local polynomial approximation to remove the demosaicking noise generated in the CDM process and then adapted this scheme to noisy CFA inputs for joint demosaicking-denoising.

The third way to remove noise from CFA data is to implement denoising before demosaicking. However, due to the underlying mosaic structure of CFAs, many existing effective monochromatic image denoising methods can not be applied to the CFA data directly. To overcome the problem, the CFA image can be divided into several sub-images using the approach known from the CFA image compression literature, e.g., [37]. Since each of the sub-images constitutes a gray-scale image, it can be enhanced using denoising algorithms from gray-scale imaging. The desired CFA image is obtained by restoring it from the enhanced sub-images. Nonetheless, such a scheme does not exploit the interchannel correlation which is essential to reduce various color shifts and artifacts in the final image [12], [13]. Since the volume of CFA images is three times less than that of the demosaicked images, there is a demand to develop new denoising algorithms which can fully exploit the interchannel correlations and operate directly on CFA images, thus achieving higher processing rates.

This paper presents a new and efficient scheme for denoising CFA images. The technique of principle component analysis (PCA) [38], [39] is employed to analyze the local structure of each CFA variable block, which contains color components from different channels. In [26], a PCA-based monochromatic image denoising scheme was proposed and here we improve the algorithm and extend it to CFA mosaic images. By adaptively computing the co-variance matrix of each variable block, the PCA could transform the noisy signal into another space, in which the signal energy is better clustered and the noise can be more effectively removed. Since there can be different and varying structures in each local training window, to improve the estimation accuracy of PCA transformation matrix we select the similar blocks to the underlying one and use them only, instead of all blocks, for PCA training. Such a training sample selection procedure can better preserve the image local structures. The proposed spatially adaptive PCA denoising scheme works directly on the CFA image and it can effectively exploit the spatial and spectral correlation simultaneously.
The rest of the paper is structured as follows. Section II briefly reviews the concept of PCA. Section III presents the PCA-based denoising algorithm for CFA images. The motivation and design characteristics are described in detail. In Section IV, experimental results are provided to demonstrate the efficiency of the proposed method for single-sensor CFA image denoising. Finally, conclusions are drawn in Section V.

\section{Principal Component Analysis (PCA)}

PCA [38], [39] is a classical de-correlation technique which has been widely used for dimensionality reduction with direct applications in pattern recognition, data compression and noise reduction. Denote by $\mathbf{x}=\left[\begin{array}{llll}x_{1} & x_{2} & \ldots & x_{m}\end{array}\right]^{T}$ an $m$-component vector variable and denote by

$$
\mathbf{X}=\left[\begin{array}{cccc}
x_{1}^{1} & x_{1}^{2} & \ldots & x_{1}^{n} \\
x_{2}^{1} & x_{2}^{2} & \ldots & x_{2}^{n} \\
\vdots & \vdots & \vdots & \vdots \\
x_{m}^{1} & x_{m}^{2} & \ldots & x_{m}^{n}
\end{array}\right]
$$

the sample matrix of $\mathbf{x}$, where $x_{i}^{j}, j=1,2, \ldots, n$, is the discrete sample of variable $x_{i}, i=1,2, \ldots, m$. The $i$ th row of sample matrix $\mathbf{X}$, denoted by $X_{i}=\left[\begin{array}{llll}x_{i}^{1} & x_{i}^{2} & \ldots & x_{i}^{n}\end{array}\right]$, is the sample vector of $x_{i}$. The mean value of $x_{i}$ can be estimated as $\mu_{i}=$ $E\left[x_{i}\right] \approx(1 / n) \sum_{j=1}^{n} X_{i}(j)$, and thus, the mean value vector of $\mathbf{x}$ is

$$
\boldsymbol{\mu}=E[\mathbf{x}]=\left[\begin{array}{llll}
\mu_{1} & \mu_{2} & \ldots & \mu_{m}
\end{array}\right]^{T} .
$$

Subtracting $\boldsymbol{\mu}$ from $\mathbf{x}$ results in the centralized vector $\overline{\mathbf{x}}=$ $\mathrm{x}-\mu$. The element of $\overline{\mathrm{x}}$ is $\bar{x}_{i}=x_{i}-\mu_{i}$ and the sample vector of $\bar{x}_{i}$ is $\bar{X}_{i}=\bar{X}_{i}-\mu_{i}=\left[\begin{array}{llll}\bar{x}_{i}^{1} & \bar{x}_{i}^{2} & \ldots & \bar{x}_{i}^{n}\end{array}\right]$, where $\bar{x}_{i}^{j}=x_{i}^{j}-\mu_{i}$. Accordingly, the centralized matrix $\overline{\mathbf{X}}$ of $\mathbf{X}$ can be expressed as follows:

$$
\overline{\mathbf{X}}=\left[\begin{array}{c}
\bar{X}_{1} \\
\bar{X}_{2} \\
\vdots \\
\bar{X}_{m}
\end{array}\right]=\left[\begin{array}{cccc}
\bar{x}_{1}^{1} & \bar{x}_{1}^{2} & \ldots & \bar{x}_{1}^{n} \\
\bar{x}_{2}^{1} & \bar{x}_{2}^{2} & \ldots & \bar{x}_{2}^{n} \\
\vdots & \vdots & \vdots & \vdots \\
\bar{x}_{m}^{1} & \bar{x}_{m}^{2} & \ldots & \bar{x}_{m}^{n}
\end{array}\right]
$$

The co-variance matrix of $\overline{\mathbf{x}}$ is calculated as $\Omega=E\left[\overline{\mathbf{x}} \overline{\mathbf{x}}^{T}\right] \approx$ $(1 / n) \overline{\mathbf{X}} \overline{\mathbf{X}}^{T}$.

The goal of PCA is to find an orthonormal transformation matrix $\mathbf{P}$ to decorrelate $\overline{\mathbf{x}}$, i.e., $\overline{\mathbf{y}}=\mathbf{P} \overline{\mathbf{x}}$ and the co-variance matrix of $\overline{\mathbf{y}}$ is diagonal. Since $\boldsymbol{\Omega}$ is symmetrical, its singular value decomposition (SVD) can be written as

$$
\Omega=\Phi \Lambda \Phi^{\mathbf{T}}
$$

where $\boldsymbol{\Phi}=\left[\begin{array}{llll}\phi_{1} & \phi_{2} & \ldots & \phi_{m}\end{array}\right]$ is the $m \times m$ orthonormal eigenvector matrix and $\boldsymbol{\Lambda}=\operatorname{diag}\left\{\lambda_{1}, \lambda_{2}, \ldots, \lambda_{m}\right\}$ is the diagonal eigenvalue matrix with $\lambda_{1} \geq \lambda_{2} \geq \cdots \geq \lambda_{m}$. The terms $\phi_{1}, \phi_{2}, \ldots, \phi_{m}$ and $\lambda_{1}, \lambda_{2}, \ldots, \lambda_{m}$ are the eigenvectors and eigenvalues of $\boldsymbol{\Omega}$. By setting

$$
\mathbf{P}=\boldsymbol{\Phi}^{\mathbf{T}}
$$

$\overline{\mathbf{X}}$ can be decorrelated, i.e., $\overline{\mathbf{Y}}=\mathbf{P} \overline{\mathbf{X}}$ and $\boldsymbol{\Lambda}=E\left[\overline{\mathbf{y}} \overline{\mathbf{y}}^{T}\right] \approx$ $(1 / n) \overline{\mathbf{Y}} \overline{\mathbf{Y}}^{T}$. 


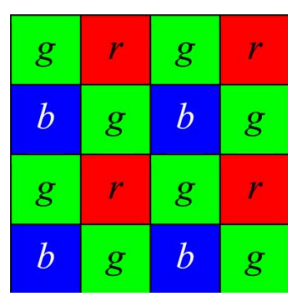

(a)

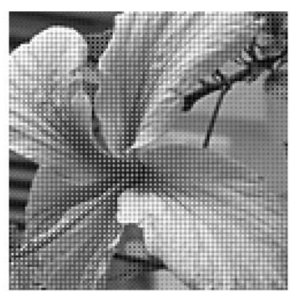

(b)

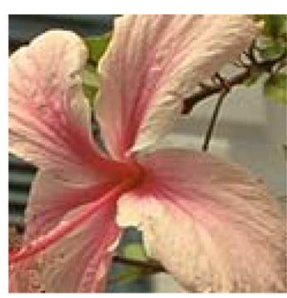

(c)
Fig. 1. Single-sensor imaging concept: (a) Bayer CFA pattern; (b) a CFA image; (c) demosaicked full-color version of (b).

Besides decorrelation, another important property of PCA is that it is optimal by using a subset of its principal components to represent the original signal. For example, one could use the first $k$ most important eigenvectors to form the transformation matrix as $\mathbf{P}^{T}=\left[\begin{array}{llll}\phi_{1} & \phi_{2} & \ldots & \phi_{k}\end{array}\right], k<m$. Then the transformed dataset $\overline{\mathbf{Y}}=\mathbf{P} \overline{\mathbf{X}}$ will be of dimension $k \times n$. Compared with the original dataset $\overline{\mathbf{X}}$, which is $m \times n$, the dimension of $\overline{\mathbf{Y}}$ is reduced while preserving most of the energy of $\overline{\mathbf{X}}$. This property is also known as optimal dimensionality reduction [39].

The optimal dimensionality reduction property of PCA can be used for noise removal. Generally speaking, the energy of a signal will concentrate on a small subset of the PCA transformed dataset, while the energy of noise will evenly spread over the whole dataset. Therefore, by preserving only the most important subset of the transformed dataset and then conducting the inverse PCA transform, the noise could be significantly reduced while the signal being well recovered. In Section III, this idea will be used to build the proposed direct CFA image denoising solution.

\section{PCA-BASED DENOISING OF CFA Mosaic IMAgES}

\section{A. CFA Sensor Noise}

Without loss of generality, the widely used Bayer pattern [1] is considered in this paper and the algorithm can be easily extended to other CFAs. In the Bayer pattern [Fig. 1(a)], the red $(r)$, green $(g)$ and blue $(b)$ samples are interlaced, with the double sampling frequency of the green channel compared to the red and blue channels. The purpose of CDM is to interpolate the two missing color components at each pixel location in the CFA image, thus restoring the full-color image from the CFA sensor readings. Fig. 1(b) shows a CFA image and Fig. 1(c) depicts the corresponding demosaicked image.

Most CDM algorithms [2]-[10], [14]-[17] operate on the assumption of noise-free CFA data. This assumption, however, is invalid in practice. Digital color imaging devices, ranging from the low cost and/or resource constrained ones (e.g., wireless camera phones) to the high-end ones (e.g., digital cinema cameras) produce images with modest to severe noise, making digital photographs not always visually pleasing. It is accepted that the corrupted noise in charge-coupled device (CCD) and complementary-symmetry/metal-oxide semiconductor (CMOS) sensors is signal-dependent [27], [35], [36]. Foi et al. [35] pointed out that the noise variance depends on the signal magnitude, while Poisson, film-grain, multiplicative and speckle models can be used to model the noise. In [27], Hirakawa modeled the raw sensor output as $y=x+\left(k_{0}+k_{1} x\right) v$, where $x$ is the desired noiseless signal, $v \in N(0,1)$ is unit Gaussian white noise and $k_{0}$ and $k_{1}$ are sensor dependent parameters. Although this noise model may fit some sensors better, the design of denoising algorithms may be complex and the computational cost may be very expensive.

One simple and widely used noise model is the signal-independent additive noise model $y=x+v$. It is a special case of the signal-dependent noise model with $k_{1}=0$ and commonly used to approximate the Poisson noise in CCD/CMOS sensors. Since the additive noise model is simple to use in the design and analysis of denoising algorithms, it has been widely used in the literature [19]-[26]. The signal-dependent noise characteristic can be compensated by estimating the noise variance adaptively in each local area [33].

In [30], Zhang et al. proposed a channel-dependent additive noise model, which is a tradeoff between the signal-dependent noise model and the signal-independent additive noise model, by considering the different types of color filters in the CFA

$$
\tilde{r}=r+v_{r}, \quad \tilde{g}=g+v_{g}, \quad \tilde{b}=b+v_{b}
$$

where $v_{r}, v_{g}$, and $v_{b}$ are the noise signals in the red, green and blue locations of the CFA image (referring to Fig. 1). The terms $r, g$, and $b$ are the desired sample values to be recovered from their noisy versions $\tilde{r}, \tilde{g}$, and $\tilde{b}$. The second order statistics of $v_{r}, v_{g}$, and $v_{b}$, i.e., the corresponding standard deviations $\sigma_{g}$, $\sigma_{r}$ and $\sigma_{b}$, may be different but the noises are assumed to be mutually uncorrelated.

We adopt the channel-dependent model in the design of the proposed CFA image denoising algorithm. It allows the noise statistics to vary in different channels because a given type of sensors behaves differently in different wavelengths. On the other hand, in channel-dependent noise model the sensor noise is independent of signal within each channel to simplify the denoising algorithm. This simplification does not materially degrade the visual quality of denoised images because the signal to noise ratio (SNR) is high anyway when the signal amplitude is high.

\section{B. CFA Block-Based Spatially Adaptive PCA}

A conventional solution to removing the effect of noise on the demosaicked full color image is to denoise after CDM. However, if noise is untreated in the demosaicking step, the noise caused color artifacts can be very hard to remove in the subsequent denoising process. The CDM process will complicate the noise characteristics by blending the noise across channels. Some joint demosaicking-denoising schemes have been reported [27], [30]. Since both demosaicking and denoising can be viewed as to estimate a sample from its neighbors, Hirakawa and Parks [27] adaptively computed a filter to accomplish the two tasks simultaneously by using the TLS technique under some constraints of the CFA pattern. Zhang et al. [30] first estimate the color difference signal from the noisy CFA image and then reconstruct the green channel by using a specific wavelet denoising algorithm. The red/blue channel is then readily recovered. Both the two schemes perform better than many demosaicking first and denoising later methods. 


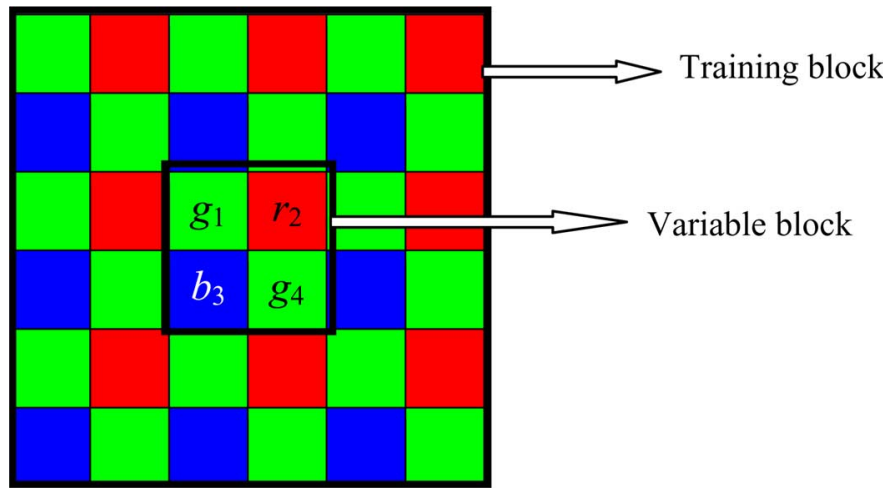

Fig. 2. Illustration of the variable block and training block in the spatially adaptive PCA-based CFA image denoising.

The other strategy to remove noise from CFA data is to implement denoising before demosaicking. Some apparent advantages of this pipeline are that it can reduce the noise-caused color artifacts, and the denoising and demosaicking algorithms can be independently designed. The difficulty of this strategy lies in the red, green and blue interlaced CFA pattern, which blocks the application of many existing effective monochromatic image denoising methods. One simple solution is to partition, for example, the big Bayer pattern CFA image into one red, one blue and two greens sub-images and then denoise them separately as gray level images. This solution, however, does not exploit the spectral correlation within red, green and blue channels.

To fully exploit the correlation among the three color channels, we propose a spatially adaptive denoising algorithm that works directly on CFA mosaic images using the PCA technique introduced in Section II. In [26], Muresan and Parks proposed a PCA-based denoising algorithm for monochromatic images. In this paper, we improve the algorithm and extend the PCA-based denoising to mosaic CFA images by considering the special structures of CFA patterns. Following the underlying layout of the CFA, such as the Bayer pattern considered throughout this paper, we define a block which consists of at least one red, one green and one blue sample. We call this block the variable block because the elements in this block will be used as the variables in PCA training. For example, Fig. 2 shows the variable block with four elements: one red, one blue and two green samples. We stretch the block to a column vector and denote it as

$$
\mathbf{x}=\left[\begin{array}{llll}
g_{1} & r_{2} & b_{3} & g_{4}
\end{array}\right]^{T} .
$$

Please note that this four-element variable block is used here for the sake of simplicity of the discussion to be followed. In practical implementations, this block can be bigger, for example, $4 \times 4,6 \times 6$, etc.

Variable vector $\mathbf{x}$ will be associated with a block which contains enough samples for training. This training block should be much bigger than the variable block in order to ensure that the statistics of the variables can be reasonably calculated. Wherever any part of the training block can match the variable block, the pixels of that part will be taken as the samples of the variable vector. For instance, in Fig. 2, there are nine samples for each element of $\mathbf{x}$. Specifically, variable $g_{1}$ is associated with green samples at locations $(1,1),(1,3),(1,5),(3,1),(3,3),(3,5),(5,1)$, $(5,3)$, and $(5,5)$. The samples of other variables can be obtained correspondingly. It is reasonable to assume that those samples are independent draws of the variable because they are acquired at different spatial locations. Let $G_{1}$ be the row vector containing all the samples associated with $g_{1}$ whereas $R_{2}, B_{3}$, and $G_{4}$ denote similar row vectors for $r_{2}, r_{3}$ and $g_{4}$, respectively. The whole dataset of $\mathbf{x}$ will be $\mathbf{X}=\left[\begin{array}{llll}G_{1}^{T} & R_{2}^{T} & B_{3}^{T} & G_{4}^{T}\end{array}\right]^{T}$.

Denote by $\mu_{g_{1}}, \mu_{r_{2}}, \mu_{b_{3}}$, and $\mu_{g_{4}}$ the mean values of variables $g_{1}, r_{2}, r_{3}$ and $g_{4}$, respectively. Those mean values can be estimated as the average of all the samples in $G_{1}, R_{2}, B_{3}$ and $G_{4}$, respectively. The mean vector of $\mathrm{x}$ can be denoted as $\boldsymbol{\mu}=\left[\begin{array}{llll}\mu_{g_{1}} & \mu_{r_{2}} & \mu_{b_{3}} & \mu_{g_{4}}\end{array}\right]^{T} . \mathbf{x}$ is centralized as $\overline{\mathbf{x}}=\mathbf{x}-\boldsymbol{\mu}$ and $\overline{\mathbf{X}}=\left[\begin{array}{llll}G_{1}^{T}-\mu_{g_{1}} & R_{2}^{T}-\mu_{r_{1}} & B_{3}^{T}-\mu_{b_{3}} & G_{4}^{T}-\mu_{g_{4}}\end{array}\right]^{T}$ is accordingly the centralized dataset of $\mathbf{X}$.

With the additive noise model, the variable of noisy observation of $\mathbf{x}$ can be expressed as follows:

$$
\tilde{\mathbf{x}}=\mathbf{x}+\mathbf{v}
$$

where $\mathbf{v}=\left[\begin{array}{llll}v_{g_{1}} & v_{r_{2}} & v_{b_{3}} & v_{g_{4}}\end{array}\right]^{T}$ is the noise variable vector. Zero mean noise characteristics dictate that the mean vector of $\tilde{\mathbf{x}}$ is the same as that of $\mathbf{x}$, i.e., $E[\tilde{\mathbf{x}}]=E[\mathbf{x}]=\boldsymbol{\mu}$. Note that, in practice, $\boldsymbol{\mu}$ is calculated from the samples of $\tilde{\mathbf{x}}$, but not $\mathbf{x}$. The centralized vector of $\tilde{\mathbf{x}}$ is then $\overline{\tilde{\mathbf{x}}}=\tilde{\mathbf{x}}-\boldsymbol{\mu}=\overline{\mathbf{x}}+\mathbf{v}$.

Similar to $\mathbf{X}$, we denote by $\mathbf{V}=\left[\begin{array}{llll}V_{g_{1}}^{T} & V_{r_{2}}^{T} & V_{b_{3}}^{T} & V_{g_{4}}^{T}\end{array}\right]^{T}$ the dataset of additive channel-dependent noise $\mathbf{v}$, where $V_{g_{1}}$ and $V_{g_{4}}$ come from green channel noise $v_{g}, V_{r_{2}}$ comes from red channel noise $v_{r}$ and $V_{b_{3}}$ from blue channel noise $v_{b}$. The available measurements of noiseless dataset $\mathbf{X}$ is then $\tilde{\mathbf{X}}=\mathbf{X}+$ $\mathbf{V}$. We subtract the mean value $\boldsymbol{\mu}$ from $\tilde{\mathbf{X}}$ to get the centralized dataset of $\overline{\tilde{\mathbf{x}}}$

$$
\overline{\tilde{\mathbf{X}}}=\overline{\mathbf{X}}+\mathbf{V}
$$

Now the problem transforms to estimate $\overline{\mathbf{X}}$ from the noisy measurement $\overline{\tilde{\mathbf{X}}}$. Suppose we have got the estimated dataset of $\overline{\mathbf{X}}$, denoted by $\hat{\mathbf{X}}$, then the samples in the training block are denoised. The central part of the training block can be extracted as the denoising block because boundary samples do not usually contribute to the denoising performance as much as the samples from the central part. The whole CFA image can be denoised by moving the denoising block from top left to bottom right. The way of removing the noise from $\overline{\tilde{\mathbf{X}}}$ using PCA techniques will be discussed in the following.

\section{Denoising in PCA Domain}

As mentioned in Section II, the optimal dimension reduction property of PCA can be used to reduce noise. By computing the covariance matrix $\boldsymbol{\Omega}_{\overline{\mathrm{x}}}$ of $\overline{\mathrm{x}}$, the optimal PCA transformation matrix $\mathbf{P}_{\overline{\mathbf{x}}}$ for $\overline{\mathbf{x}}$ can be obtained by using (2-4) and (2-5). However, the available dataset $\overline{\mathbf{X}}$ is noise corrupted so that $\boldsymbol{\Omega}_{\overline{\mathrm{X}}}$ cannot be directly computed. Fortunately, $\boldsymbol{\Omega}_{\overline{\mathrm{x}}}$ can be estimated using the linear noise model $\overline{\tilde{\mathbf{x}}}=\overline{\mathbf{x}}+\mathbf{v}$. 
Assuming that $n$ training samples are available for each element of $\overline{\mathbf{x}}$, the covariance matrix of $\overline{\tilde{\mathbf{x}}}$ can be estimated using maximal likelihood estimation (MLE)

$$
\begin{aligned}
\mathbf{\Omega}_{\overline{\mathbf{x}}} & =E\left[(\overline{\tilde{\mathbf{x}}}-E[\overline{\tilde{\mathbf{x}}}])(\overline{\tilde{\mathbf{x}}}-E[\overline{\mathbf{x}}])^{T}\right] \\
& \approx \frac{1}{n} \overline{\tilde{\mathbf{X}}} \overline{\tilde{\mathbf{X}}}^{T} \\
& =\frac{1}{n}\left(\overline{\mathbf{X}} \overline{\mathbf{X}}^{T}+\mathbf{\mathbf { X }} \mathbf{V}^{T}+\mathbf{V} \overline{\mathbf{X}}^{T}+\mathbf{V} \mathbf{V}^{T}\right) .
\end{aligned}
$$

Since the signal $\overline{\mathbf{X}}$ and noise $\mathbf{V}$ are uncorrelated, items $\overline{\mathbf{X}} V^{T}$ and $\mathbf{V} \overline{\mathbf{X}}^{T}$ will be nearly zero matrices which reduces the above expression of $\boldsymbol{\Omega}_{\overline{\tilde{\mathrm{x}}}}$ to the following:

$$
\boldsymbol{\Omega}_{\overline{\tilde{\mathbf{x}}}}=\boldsymbol{\Omega}_{\overline{\mathbf{x}}}+\boldsymbol{\Omega}_{\mathbf{V}} \approx \frac{1}{n}\left(\overline{\mathbf{X}} \overline{\mathbf{X}}^{T}+\mathbf{V} \mathbf{V}^{T}\right)
$$

where $\boldsymbol{\Omega}_{\overline{\mathrm{x}}} \approx(1 / n) \overline{\mathbf{X}} \overline{\mathbf{X}}^{T}$ and $\boldsymbol{\Omega}_{\mathbf{V}} \approx(1 / n) \mathbf{V} \mathbf{V}^{T}$ are the covariance matrices of $\overline{\mathbf{X}}$ and $\mathbf{v}$, respectively.

Estimating $\boldsymbol{\Omega}_{\overline{\mathrm{x}}}$ from $\boldsymbol{\Omega}_{\overline{\mathrm{x}}}$ requires $\boldsymbol{\Omega}_{\mathbf{V}}$ is known. With the noise vector $\mathbf{v}=\left[\begin{array}{llll}v_{g_{1}} & v_{r_{2}} & v_{b_{3}} & v_{g_{4}}\end{array}\right]^{T}$ and the fact that the four elements of $\mathbf{v}$ are uncorrelated with each other, we have

$$
\mathbf{\Omega}_{\mathbf{V}}=E\left[\mathbf{v v}^{T}\right]=\operatorname{diag}\left\{\sigma_{g}^{2}, \sigma_{r}^{2}, \sigma_{b}^{2}, \sigma_{g}^{2}\right\}
$$

where $\sigma_{g}, \sigma_{r}$, and $\sigma_{b}$ are the standard deviations of the channeldependent noise $v_{g}, v_{r}$ and $v_{b}$ in (3-1). With (3-6) and (3-7), the covariance of $\overline{\mathbf{x}}$ can be calculated as $\boldsymbol{\Omega}_{\overline{\mathrm{x}}}=\boldsymbol{\Omega}_{\overline{\mathrm{x}}}-\boldsymbol{\Omega}_{\mathrm{V}}$. Thus, there can be some negative values of $\boldsymbol{\Omega}_{\overline{\mathrm{x}}}$ in the diagonal positions. In the implementation, we will replace the negative values with zero or a small positive number, e.g., 0.0001 .

Now we can decompose $\boldsymbol{\Omega}_{\overline{\mathrm{x}}}$ by using (2-4) as

$$
\Omega_{\overline{\mathrm{x}}}=\Phi_{\overline{\mathrm{x}}} \Lambda_{\overline{\mathrm{x}}} \Phi_{\overline{\mathrm{x}}}^{\mathrm{T}}
$$

where $\boldsymbol{\Phi}_{\overline{\mathbf{x}}}=\left[\begin{array}{llll}\phi_{1} & \phi_{2} & \phi_{3} & \phi_{4}\end{array}\right]$ is the $4 \times 4$ orthonormal eigenvector matrix and $\Lambda_{\overline{\mathbf{x}}}=\operatorname{diag}\left\{\lambda_{1}, \lambda_{2}, \lambda_{3}, \lambda_{4}\right\}$ is the diagonal eigenvalue matrix with $\lambda_{1} \geq \lambda_{2} \geq \lambda_{3} \geq \lambda_{4}$. The orthonormal PCA transformation matrix for $\overline{\overline{\mathbf{X}}}$ is then

$$
\mathbf{P}_{\overline{\mathrm{x}}}=\boldsymbol{\Phi}_{\overline{\mathrm{x}}}^{\mathbf{T}} .
$$

It should be noted that if the noise levels of $v_{g}, v_{r}$, and $v_{b}$ are the same, i.e., $\sigma_{g}=\sigma_{r}=\sigma_{b}$, then $\boldsymbol{\Omega}_{\mathrm{V}}$ will be an identity matrix with a scaling factor $\sigma_{g}^{2}$. In this case, it can be proved that the SVD of $\boldsymbol{\Omega}_{\overline{\mathrm{x}}}$ and $\boldsymbol{\Omega}_{\overline{\mathrm{x}}}$ will give the same eigenvector matrix $\boldsymbol{\Phi}_{\overline{\mathrm{x}}}$ and, hence, the same PCA transformation matrix $\mathbf{P}_{\overline{\mathbf{x}}}$. However, when $\sigma_{g}, \sigma_{r}$, and $\sigma_{b}$ are different, $\boldsymbol{\Omega}_{\mathbf{V}}$ can not be scaled to an identity matrix and, therefore, $\boldsymbol{\Omega}_{\overline{\tilde{x}}}$ and $\boldsymbol{\Omega}_{\overline{\mathrm{x}}}$ will yield different eigenvector matrices by SVD.

Applying $\mathbf{P}_{\overline{\mathbf{x}}}$ to the noisy dataset $\overline{\tilde{\mathbf{X}}}$ resulting in the following:

$$
\begin{aligned}
\overline{\tilde{\mathbf{Y}}} & =\mathbf{P}_{\overline{\mathbf{x}}} \overline{\tilde{\mathbf{X}}}=\mathbf{P}_{\overline{\mathbf{x}}}(\overline{\mathbf{X}}+\mathbf{V}) \\
& =\mathbf{P}_{\overline{\mathbf{x}}} \overline{\mathbf{X}}+\mathbf{P}_{\overline{\mathbf{x}}} \mathbf{V}=\overline{\mathbf{Y}}+\mathbf{V}_{\mathbf{Y}}
\end{aligned}
$$

where $\overline{\mathbf{Y}}=\mathbf{P}_{\overline{\mathbf{x}}} \overline{\mathbf{X}}$ is the decorrelated dataset for signal and $\mathbf{V}_{\mathbf{Y}}=\mathbf{P}_{\overline{\mathbf{x}}} \mathbf{V}$ is the transformed noise dataset for noise. Since signal $\overline{\mathbf{Y}}$ and noise $\mathbf{V}_{\mathbf{Y}}$ are uncorrelated, the covariance matrix of $\overline{\tilde{Y}}$ is

$$
\Omega_{\overline{\mathbf{y}}}=\Omega_{\overline{\mathbf{y}}}+\Omega_{\mathbf{v}_{\mathbf{y}}} \approx \frac{1}{n} \overline{\tilde{\mathbf{Y}}} \overline{\tilde{\mathbf{Y}}}^{T}
$$

where

$$
\begin{aligned}
\boldsymbol{\Omega}_{\overline{\mathbf{y}}} & =\boldsymbol{\Lambda}_{\overline{\mathbf{x}}} \approx \frac{1}{n} \overline{\mathbf{Y}} \overline{\mathbf{Y}} T \\
\boldsymbol{\Omega}_{\mathrm{v}_{\mathbf{y}}} & =\mathbf{P}_{\overline{\mathbf{x}}} \boldsymbol{\Omega}_{\mathbf{v}} \mathbf{P}_{\overline{\mathbf{x}}}^{T} \approx \frac{1}{n} \mathbf{V}_{\mathbf{Y}} \mathbf{V}_{\mathbf{Y}}^{T}
\end{aligned}
$$

are the covariance matrices of $\overline{\mathbf{Y}}$ and $\mathbf{V}_{\mathbf{Y}}$, respectively.

In the PCA transformed domain $\overline{\mathbf{Y}}$ in (3-10), most energy of $\overline{\mathbf{Y}}$ concentrates on the several most important components, i.e., the first several rows of $\overline{\tilde{Y}}$ whereas the energy of noise $\mathbf{V}_{\mathbf{Y}}$ is distributed in $\overline{\tilde{Y}}$ much more evenly. Therefore, resetting the last several rows (the least important components) of $\overline{\tilde{Y}}$ as zeros will preserve well the signal $\overline{\mathbf{Y}}$ while removing the noise $\mathbf{V}_{\mathbf{Y}}$. This operation is actually based on the optimal dimension reduction property of PCA, as discussed in Section II. We denote by $\overline{\tilde{\mathbf{Y}}}^{d}$ the dimension reduced (by resetting the last several rows as zeros) dataset of $\overline{\tilde{\mathbf{Y}}}$, and $\overline{\tilde{\mathbf{Y}}}^{d}$ can be written as $\overline{\tilde{\mathbf{Y}}}^{d}=\overline{\mathbf{Y}}^{d}+\mathbf{V}_{\mathbf{Y}}^{d}$, where $\overline{\mathbf{Y}}^{d}$ and $\mathbf{V}_{\mathbf{Y}}^{d}$ represent, respectively, the dimension reduced datasets of $\overline{\mathbf{Y}}$ and $\mathbf{V}_{\mathbf{Y}}$. Similarly, the corresponding covariance matrices, denoted as $\boldsymbol{\Omega}_{\tilde{\mathbf{y}}}^{d}, \boldsymbol{\Omega}_{\overline{\mathbf{y}}}^{d}$, and $\boldsymbol{\Omega}_{\mathbf{v}_{\mathbf{y}}}^{d}$, are related as $\boldsymbol{\Omega}_{\tilde{\mathbf{y}}}^{d}=\boldsymbol{\Omega}_{\overline{\mathbf{y}}}^{d}+\boldsymbol{\Omega}_{\mathbf{v}_{\mathbf{y}}}^{d}$.

The noise in dimension reduced dataset $\overline{\tilde{\mathbf{Y}}}^{d}$ can be further suppressed by using linear minimum mean squared-error estimation (LMMSE). The LMMSE of $\overline{\mathbf{Y}}_{i}^{d}$, i.e., the $i$ th row of $\overline{\mathbf{Y}}^{d}$, is obtained as

$$
\hat{\overline{\mathbf{Y}}}_{i}^{d}=c_{i} \cdot \overline{\tilde{\mathbf{Y}}}_{i}^{d}
$$

where $c_{i}=\boldsymbol{\Omega}_{\overline{\mathbf{y}}}^{d}(i, i) /\left(\boldsymbol{\Omega}_{\overline{\mathbf{y}}}^{d}(i, i)+\boldsymbol{\Omega}_{\mathbf{v}_{\mathbf{y}}}^{d}(i, i)\right)$. Applying (3-14) to each nonzero row of $\overline{\mathbf{Y}}^{d}$ yields the full denoised dataset $\hat{\overline{\mathbf{Y}}}^{d}$. Now, the denoised result of the original dataset $\overline{\tilde{X}}$, i.e., the estimation of unknown noiseless dataset $\overline{\mathbf{X}}$, can be obtained by transforming back $\hat{\overline{\mathbf{Y}}}^{d}$ from PCA domain to time domain as follows:

$$
\hat{\overline{\mathbf{X}}}=\mathbf{P}_{\overline{\mathbf{X}}}^{-1} \cdot \hat{\overline{\mathbf{Y}}}^{d} .
$$

Reformatting $\hat{\bar{X}}$ results in the denoised CFA block.

In the proposed PCA-based CFA image denoising algorithm, we need to set two parameters: the sizes of variable block and training block. (The denoising block can be set as the same as or smaller than the variable block.) Different settings of the two parameters lead to different results and have different complexity. If the resolution of the image is low, the size of the variable block should be relatively small because the spatial correlation of low resolution images will also be low. Empirically, we found that setting the variable block as $4 \times 4,6 \times 6$ or $8 \times 8$ can achieve good results for most of the testing images. The size of training block can be 16 (or higher) times that of the variable block, e.g., $24 \times 24$ or $30 \times 30$ for a $6 \times 6$ variable block. 


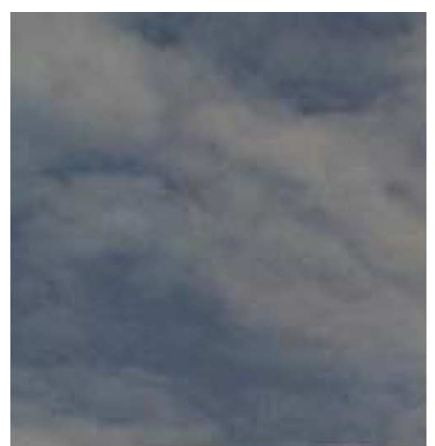

$(\mathrm{a}-1)$

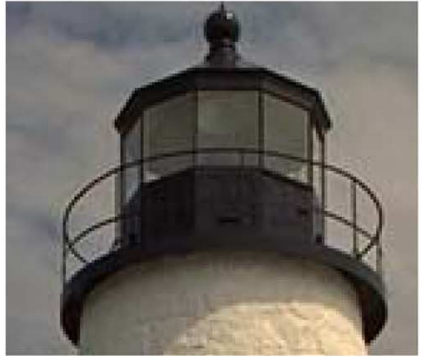

(b-1)

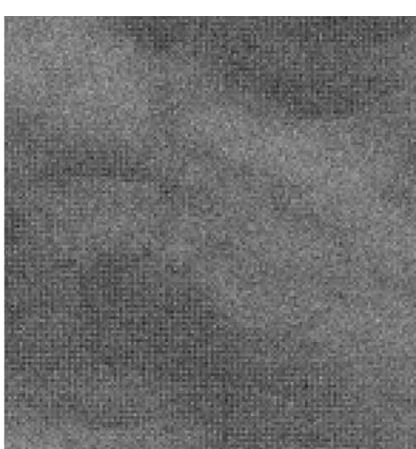

$(\mathrm{a}-2)$

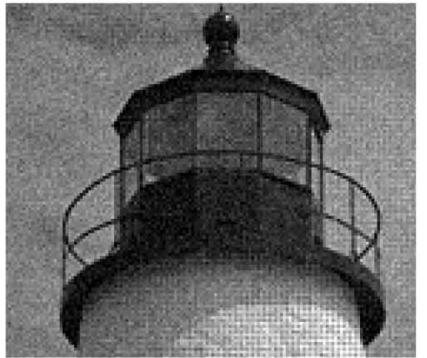

$(b-2)$

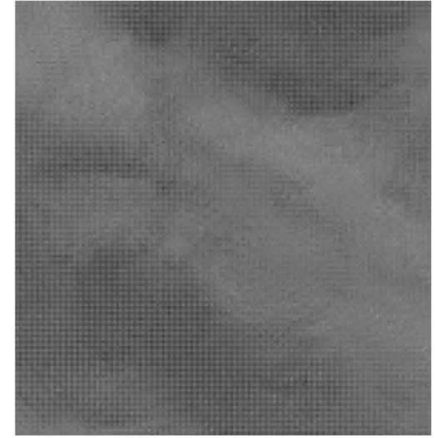

$(\mathrm{a}-3)$

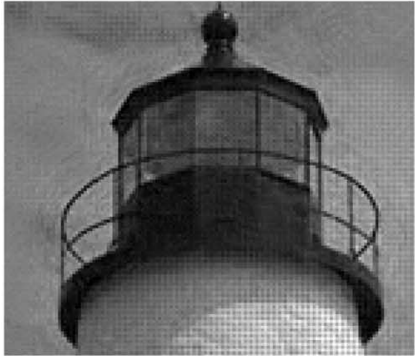

(b-3)

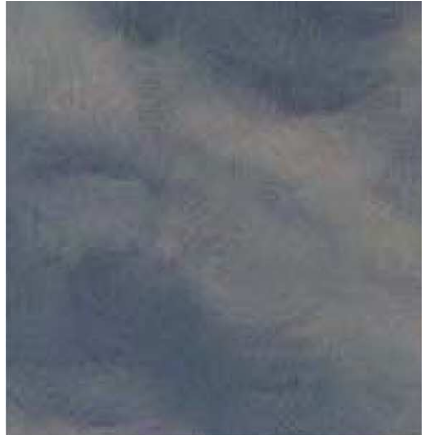

$(\mathrm{a}-4)$

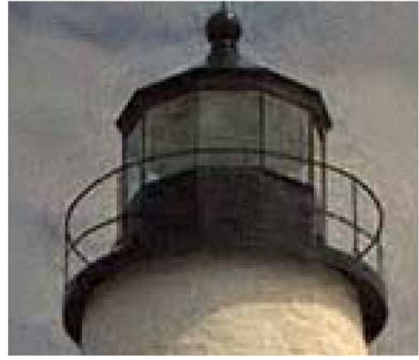

(b-4)

Fig. 3. First row, left to right: Smooth area of an original image; the noisy CFA image of it; the PCA-based denoising result and the finally demosaicked image. Similarly, the second row shows an example of the phantom artifacts along edge boundaries with smooth background.

\section{CFA Image Decomposition and Training Sample Selection}

The PCA-based denoising scheme described in the previous sections can be directly applied to the noisy CFA image for noise suppression. Although the noise can be effectively removed, in the experiments we found two problems of the proposed method. First there can be some visible noise residual in the smooth areas. Second there can be some phantom artifacts along edge boundaries with smooth background. Fig. 3(a) and (b) shows two examples respectively. In the first row, Fig. 3 (a-1)-(a-4) shows the cropped smooth area of an original image, the noisy CFA image of it, the denoised CFA image by the proposed PCA-based denoising scheme and the demosaicking result of the denoised CFA (the demosaicking scheme [9] is used here), respectively. In the second row, Fig. 3 (b-1)-(b-4) shows an example of the phantom artifacts along the boundary between edges and smooth background. Such noise residual and phantom artifacts make the reconstructed color image visually unpleased in some areas.

The noise residual in the smooth areas is mainly caused by the relatively low local signal to noise ratio or contrast. Since there are no strong edge structures in the smooth areas, there are fewer significant principal components in the PCA domain, and, hence, the discrimination between noise and signals is not as effective as that in the areas with strong edges. In addition, the training samples for the red, green and blue variables are collected from the local window according to the Bayer pattern. The mean values of the variables are computed as the average of the samples and then subtracted from the sample matrix for covariance calculation. However, in this way, the neighboring red and green pixels will not contribute to the mean value estimation of the blue variable, and so do for red and green variables. The biases in mean value estimation will lead to estima- tion bias of co-variance matrix and, hence, the PCA transformation matrix.

The reason for the phantom artifacts along edge boundaries with smooth background is as follows. The proposed PCA-based denoising algorithm will use a local training block to estimate the PCA transformation matrix. All the possible samples in the training block are used in the calculation. However, sample structures may change within a block, especially if the block contains object boundaries with smooth background. Involving such samples in the PCA training may lead to much bias in the estimation of PCA transformation matrix and consequently reduce the denoising performance, e.g., generating many phantom artifacts.

To overcome the above two problems, we propose two preprocessing steps before applying the PCA-based denoising. First, we decompose the noisy CFA image into two parts: the low-pass smooth image and the high-pass image. Denote by $I_{v}$ the noisy CFA image. We use a 2-D Gaussian low-pass filter $G(x, y)=1 / \sqrt{2 \pi} \operatorname{sexp}\left(-x^{2}+y^{2} / 2 s^{2}\right)$ to smooth $I_{v}$

$$
I_{v}^{l}=I_{v} * G \text {. }
$$

The high-pass image is then obtained as

$$
I_{v}^{h}=I_{v}-I_{v}^{l} \text {. }
$$

With a suitable scale parameter $s$ in the Gaussian filter, the low-pass image $I_{v}^{l}$ will be almost noiseless and most of the noise is contained in the high-pass output $I_{v}^{h}$, which also contains the image edge structures to be preserved. Since $I_{v}^{l}$ is almost noiseless, we do not make further processing on it. The PCA-based CFA denoising scheme will be applied to the high-pass image $I_{v}^{h}$, where the noise will be dominant in the smooth areas and they can then be better suppressed by LMMSE filtering in the 


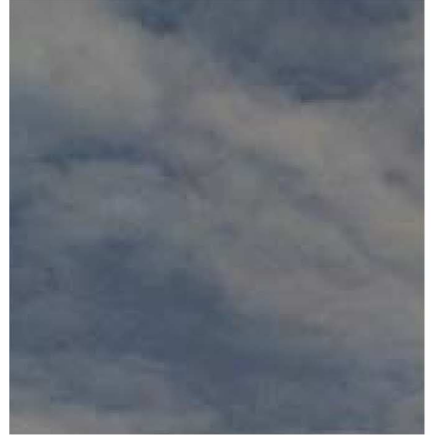

(a-1)

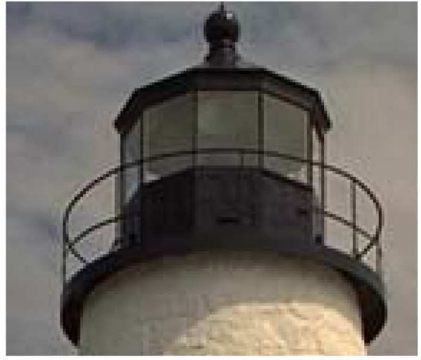

(b-1)

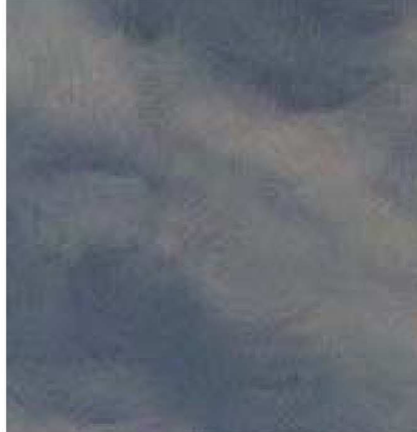

(a-2)

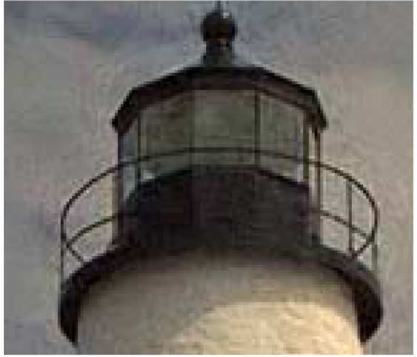

(b-2)

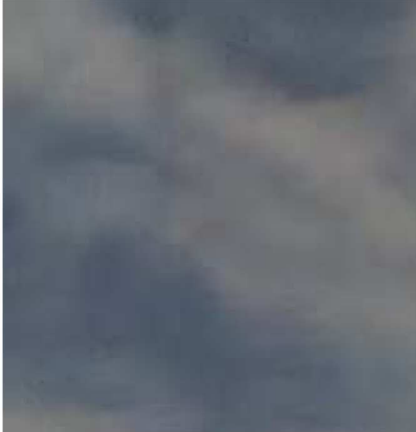

(a-3)

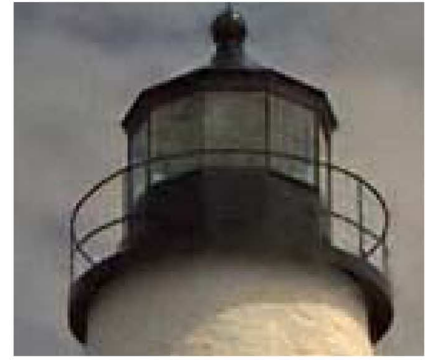

(b-3)

Fig. 4. First row, from left to right: Smooth area of an original image; the denoised and demosaicked image without image decomposition and training sample selection; the denoised and demosaicked image with image decomposition and training sample selection. Similarly, the second row shows the results on the boundary between edges and smooth background.

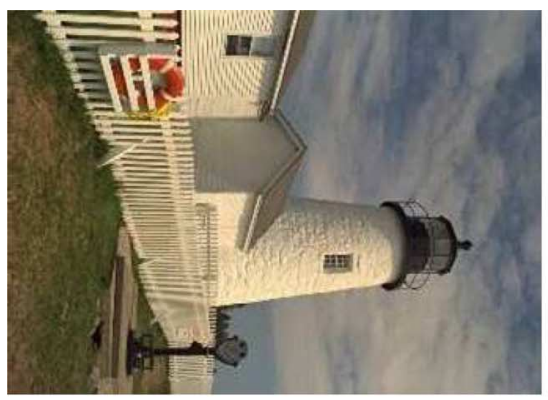

(a)

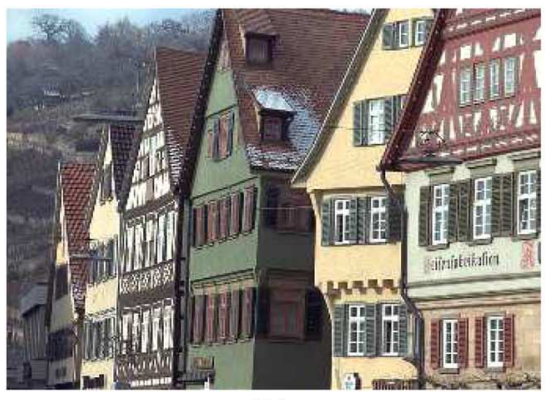

(b)

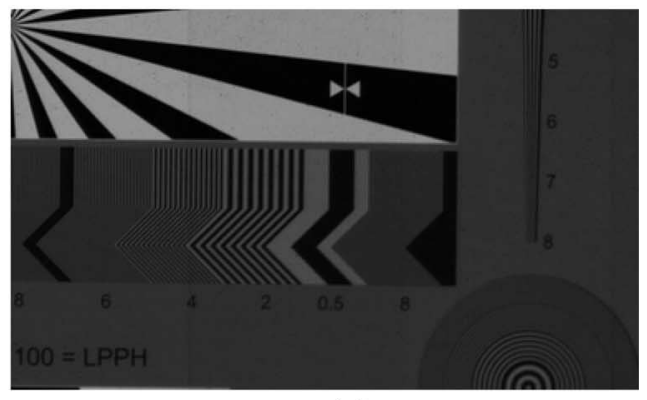

(c)

Fig. 5. Images used in the experiment. (a) Test image fence; (b) test image houses; (c) a real CFA image resolution captured by single-sensor camera.

PCA domain. Denote by $\hat{I}_{v}^{h}$ the denoised image of $I_{v}^{h}$, the final denoised CFA image is obtained as $\hat{I}=I_{v}^{l}+\hat{I}_{v}^{h}$. It can be validated that in a local window of $I_{v}^{h}$, the mean value of red, green or blue variable will be nearly zero for smooth areas. In some sense, the Gaussian smoothing operation can be viewed as a procedure to better estimate the mean values of red, green and blue variables so that the noise residual in smooth areas can be reduced effectively.

Now let's focus on how to reduce the phantom artifacts around edge boundaries with smooth background. As mentioned before, such artifacts are caused by the inappropriate training samples in the training block. Intuitively, one solution to this problem is to select the similar blocks to the underlying variable block and use them only but not all the blocks for PCA training. Such a training sample selection procedure can better estimate the co-variance matrix of the variable block and, hence, lead to a more accurate PCA transformation matrix.
Finally, image local edge structures can be better preserved by removing the phantom artifacts.

Denote by $\tilde{\mathbf{X}}$ the variable block in $I_{v}^{h}$ and by $\tilde{\mathbf{X}}$ the associated training dataset generated from the training block centered on $\tilde{\mathbf{X}}$ (refer to Section III-B). Each column of matrix $\tilde{\mathbf{X}}$ can be a sample vector of $\tilde{\mathbf{x}}$. In the method described in Section III-C, the whole dataset $\tilde{\mathbf{X}}$ is used for training. Here we select the best samples from $\tilde{\mathbf{X}}$ for PCA transformation. Denote by $\vec{x}_{k}$, $k=1,2, \ldots, n$, the $k$ th column of $\tilde{\mathbf{X}}$, and denote by $\vec{x}_{0}$ the sample vector containing the samples at the variable block $\tilde{\mathbf{x}}$. The noiseless counterparts of $\vec{x}_{k}$ and $\vec{x}_{0}$ are denoted as $\vec{x}_{k}$ and $\vec{x}_{0}$, respectively. The length of vectors $\vec{x}_{k}$ is $m$. The $L_{2}$ distance between $\vec{x}_{0}$ and $\vec{x}_{k}$ can be computed as

$$
d_{k}=\frac{1}{m} \sum_{i=1}^{m}\left(\vec{x}_{k}(i)-\vec{x}_{0}(i)\right)^{2} \approx \frac{1}{m} \sum_{i=1}^{m}\left(\vec{x}_{k}(i)-\vec{x}_{0}(i)\right)^{2}+\sigma_{a}^{2}
$$




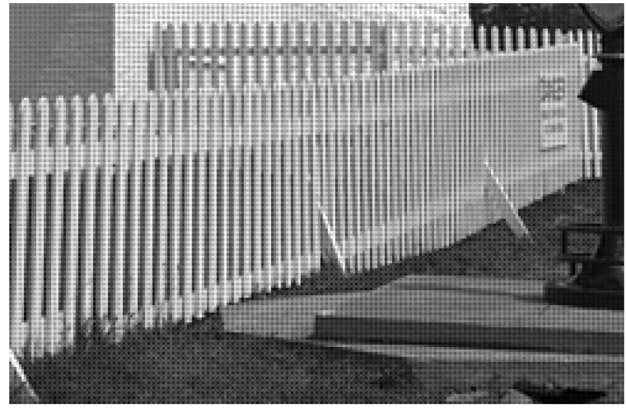

(a)

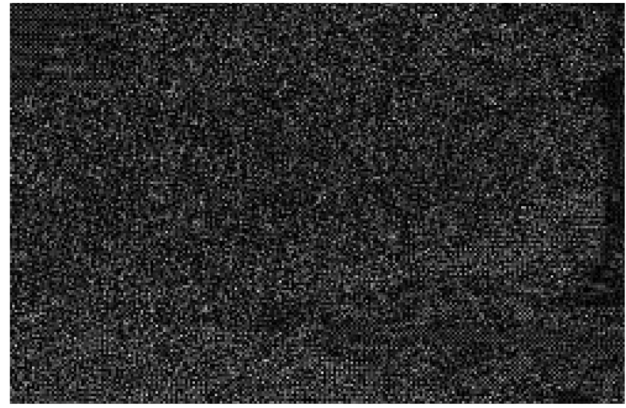

(c)

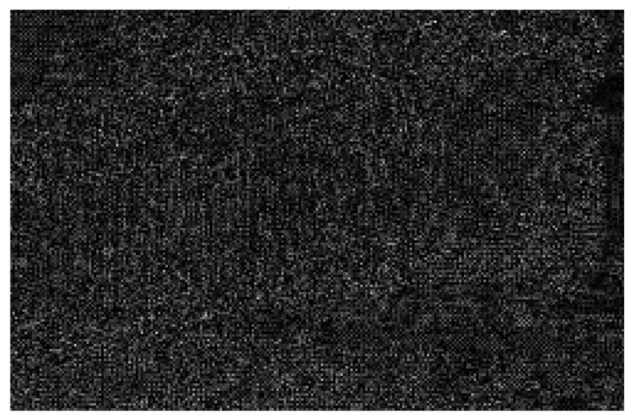

(e)

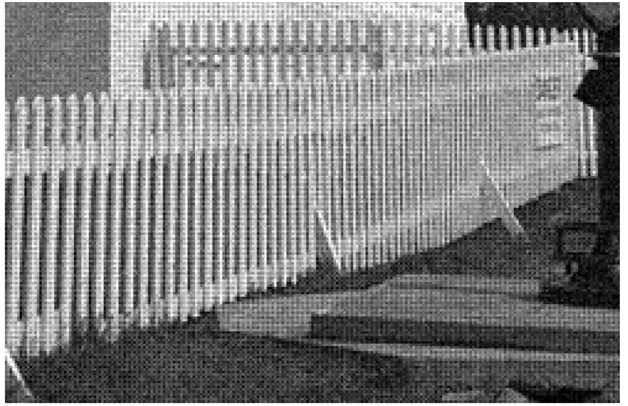

(b)

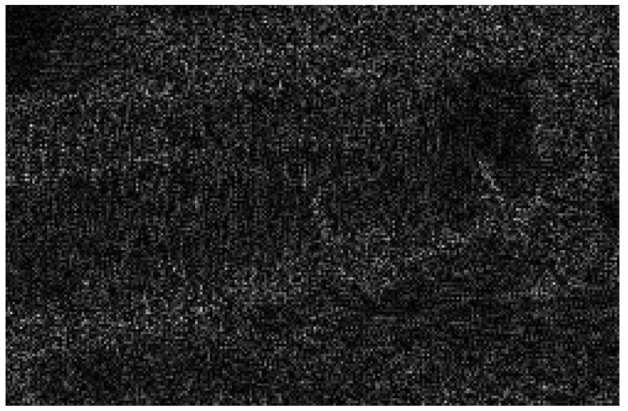

(d)

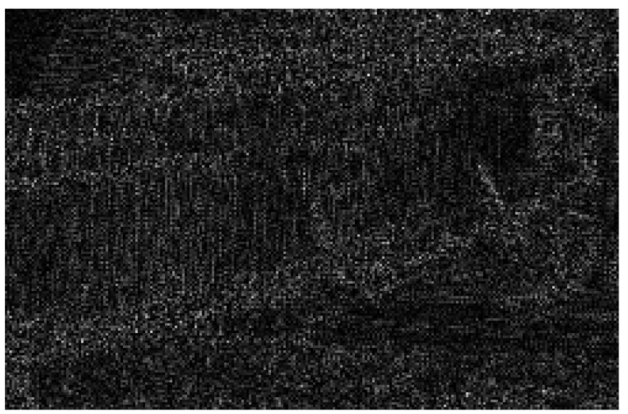

(f)

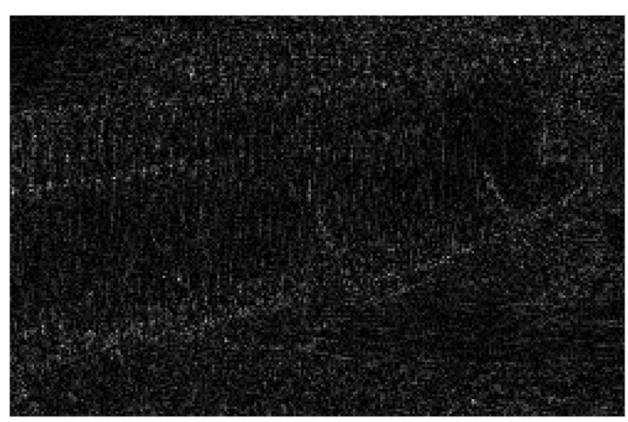

(g)

Fig. 6. Denoising results of CFA image "fence." (a) Original CFA image; (b) noisy CFA image ( $\left.\sigma_{r}=13, \sigma_{g}=12, \sigma_{b}=10\right)$; difference between the denoised and original CFA images by using methods (c) in [24] (as a whole image, PSNR $=28.80 \mathrm{~dB}$ ); (d) in [24] (as four sub-images, PSNR $=30.86 \mathrm{~dB}$ ); (e) in [25] (as a whole image, PSNR = 30.03 dB); (f) in [25] (as four sub-images, PSNR $=30.96 \mathrm{~dB}$ ); and (g) the proposed adaptive PCA-based CFA denoising method $(\mathrm{PSNR}=31.78 \mathrm{~dB})$.

where $\sigma_{a}=(1 / 2) \sqrt{\sigma_{r}^{2}+2 \sigma_{g}^{2}+\sigma_{b}^{2}}$. Obviously, the smaller the distance $d_{k}$ is, the more similar $\vec{x}_{k}$ is to $\vec{x}_{0}$.

Therefore, we select the training samples $\vec{x}_{k}$ based on its associated distance $d_{k}$. If

$$
d_{k} \leq T^{2}+\sigma_{a}^{2}
$$

then $\vec{x}_{k}$ will be selected as one training sample of $\tilde{\mathbf{x}}$, where $T$ is a preset threshold and it can be set about 5 in implementation. Suppose totally $K$ samples are selected. In practice, we need a large enough $K$ to guarantee a reasonable estimation of covariance matrix of $\tilde{\mathbf{x}}$. Therefore, if $K<100$, we will use the first $100 \vec{x}_{k}$ that give the smallest distance $d_{k}$ as the training samples. Denote by $\tilde{\mathbf{X}}_{b}$ the dataset composed of the selected $K$ sample 
TABLE I

PSNR (DB) REsults of THE CFA IMAGES By DifFERENT DENOISING METHODS

\begin{tabular}{|c|c|c|c|c|}
\hline \multirow{2}{*}{ Images } & \multirow{2}{*}{\multicolumn{2}{|c|}{ Denoising Methods }} & \multicolumn{2}{|c|}{ PSNR Results (dB) } \\
\hline & & & $\sigma_{r}=\sigma_{g}=\sigma_{b}=12$ & $\sigma_{r}=13, \sigma_{g}=12, \sigma_{b}=10$ \\
\hline \multirow{5}{*}{ Fence } & \multirow{2}{*}[24]{} & as a whole image & 28.72 & 28.80 \\
\hline & & as four sub-images & 30.76 & 30.86 \\
\hline & \multirow{2}{*}[25]{} & as a whole image & 29.90 & 30.03 \\
\hline & & as four sub-images & 30.86 & 30.96 \\
\hline & \multicolumn{2}{|c|}{ PCA-based CFA Denoising } & 31.71 & 31.78 \\
\hline \multirow{5}{*}{ House } & \multirow{2}{*}{ [24] } & as a whole image & 28.17 & 28.24 \\
\hline & & as four sub-images & 28.62 & 28.77 \\
\hline & \multirow{2}{*}[25]{} & as a whole image & 28.82 & 28.92 \\
\hline & & as four sub-images & 29.01 & 29.14 \\
\hline & \multicolumn{2}{|c|}{ PCA-based CFA Denoising } & 29.67 & 29.75 \\
\hline
\end{tabular}

vectors $\vec{x}_{k}$. Then the algorithms described in Section III-C will be applied to $\tilde{\mathbf{X}}_{b}$, instead of the original dataset $\tilde{\mathbf{X}}$.

Fig. 4 compares the denoising results by the methods with and without the procedures of CFA image decomposition and training sample selection. The first row shows the results on the smooth area and the second row shows the results on the boundary between edges and smooth background. We can clearly see that the procedures of image decomposition and training sample selection can remarkably improve the visual quality of the denoised and demosaicked images.

\section{E. Summary of the Algorithm}

The proposed spatially adaptive PCA-based CFA denoising algorithm is summarized as follows.

1. Estimate the noise standard deviations $\sigma_{g}, \sigma_{r}$ and $\sigma_{b}$ of the red, green and blue channels. (Referring to Section IV-C for the noise estimation of CFA images.)

2. Decompose the noisy CFA image $I_{v}$ into $I_{v}^{l}$ and $I_{v}^{h}$ using (3-16) and (3-17). Apply the following denoising steps 3 and 4 to $I_{v}^{h}$.

3.Set the sizes of variable block and training block. The noise co-variance matrix $\Omega_{\mathrm{V}}$ can then be determined.

4. For each training block:

Perform the training sample selection procedure (refer to

Section III-D).

Denote by $\overline{\mathbf{X}}$ the selected training dataset.

Calculate the co-variance matrix $\boldsymbol{\Omega}_{\bar{x}}$ using (3-5);

Estimate the co-variance matrix of signal as $\boldsymbol{\Omega}_{\overline{\mathrm{x}}}=\boldsymbol{\Omega}_{\overline{\mathrm{x}}}-\boldsymbol{\Omega}_{\mathrm{V}}$;

Factorize $\boldsymbol{\Omega}_{\overline{\mathrm{x}}}=\boldsymbol{\Phi}_{\overline{\mathrm{x}}} \boldsymbol{\Lambda}_{\overline{\mathrm{x}}} \boldsymbol{\Phi}_{\overline{\mathrm{x}}}^{\mathbf{T}}$ using (3-8) and set the PCA transformation matrix $\mathbf{P}_{\overline{\mathrm{x}}}=\boldsymbol{\Phi}_{\overline{\mathrm{x}}}^{\mathrm{T}}$;

Transform the dataset to PCA domain: $\overline{\tilde{\mathbf{Y}}}=\mathbf{P}_{\overline{\mathbf{x}}} \overline{\tilde{\mathbf{X}}}$;

By resetting the last several rows of $\overline{\tilde{\mathbf{Y}}}$ to zeros, reduce $\overline{\tilde{\mathbf{Y}}}$ to $\overline{\tilde{\mathbf{Y}}}^{d}$ (dimension reduction);

Shrink each row of $\overline{\tilde{\mathbf{Y}}}^{d}$ as $\hat{\overline{\mathbf{Y}}}_{i}^{d}=c_{i} \cdot \overline{\tilde{\mathbf{Y}}}_{i}^{d}$ using (3-14);
Transform back $\hat{\overline{\mathbf{Y}}}^{d}$ to time domain as $\hat{\overline{\mathbf{X}}}=\mathbf{P}_{\overline{\mathbf{X}}}^{-1} \cdot \hat{\overline{\mathbf{Y}}}^{d}$; Reformat $\hat{\overline{\mathbf{X}}}$ to get the denoised CFA block.

End

5. Denote by $\hat{I}_{v}^{h}$ the denoised output of $I_{v}^{h}$, the final denoised image is $\hat{I}=I_{v}^{l}+\hat{I}_{v}^{h}$.

\section{EXPERIMENTAL RESULTS}

To test comprehensively the performance of the proposed PCA-based CFA denoising algorithm, we carry out extensive experiments in this section. Three different types of experiments are made. First, we evaluate its denoising outputs in comparison with those by applying other denoising schemes to CFA images. Second, joint assessment of the proposed CFA denoising scheme with demosaicking schemes is carried out in comparison with many "demosaicking first and denoising later" and "joint denoising and demosaicking" algorithms. At last, a real raw CFA image is used to illustrate the performance of the proposed method. In the following, we report those experimental results in details.

\section{A. Assessment on CFA Denoising Result}

Let's first evaluate the proposed method on denoising CFA images. Two sophisticated wavelet-based denoising techniques [24], [25] were used for comparison. ${ }^{1}$ Since the two denoising schemes were designed for monochromatic images, but not for CFA mosaic images, we tested them by two scenarios in the experiments. In the first scenario, we viewed the CFA images as monochromatic images and applied the denoising schemes [24], [25] directly to them. In the second scenario, we partitioned a CFA image into four sub-images (i.e., one red, one blue and two green channel sub-images) and used them for denoising. In the second scenario, each sub-image is a true monochromatic image. In the experiments, the proposed method uses the variable and denoising blocks of $6 \times 6$ pixels and the training block of $30 \times 30$ pixels. In CFA image decomposition and training sample selection, the scale of Gaussian smooth filter is set as $s=3$ and the threshold is set as $T=5$.

The performance of the above solutions has been examined using a variety of test images, with examples depicted

${ }^{1}$ We thank the authors of [24] and [25] for sharing their codes. 
TABLE II

PSNR (DB) Results of the ReConstructed FENCE IMAGES by DifFERENT DEMOSAICKING AND Denoising Methods

\begin{tabular}{|c|c|c|c|c|c|c|c|}
\hline \multirow{3}{*}{ Demosaicking Methods } & \multirow{3}{*}{ Denoising Methods } & \multicolumn{6}{|c|}{ PSNR Results (dB) } \\
\hline & & \multicolumn{3}{|c|}{$\sigma_{r}=\sigma_{g}=\sigma_{b}=12$} & \multicolumn{3}{|c|}{$\sigma_{r}=13, \sigma_{g}=12, \sigma_{b}=10$} \\
\hline & & $\mathrm{R}$ & G & B & $\mathrm{R}$ & G & $\mathrm{B}$ \\
\hline \multirow{2}{*}{ [4] } & [24] & 28.0 & 30.3 & 28.8 & 28.4 & 30.3 & 28.6 \\
\hline & [25] & 28.1 & 30.6 & 28.3 & 27.9 & 30.6 & 28.6 \\
\hline \multirow{2}{*}{ [9] } & [24] & 30.5 & 31.3 & 30.8 & 30.3 & 31.3 & 31.2 \\
\hline & [25] & 30.5 & 31.3 & 30.9 & 30.3 & 31.3 & 31.2 \\
\hline \multirow{2}{*}{ [10] } & {$[24]$} & 29.9 & 30.7 & 30.1 & 29.7 & 30.7 & 30.3 \\
\hline & [25] & 30.2 & 31.1 & 30.6 & 30.0 & 31.2 & 30.9 \\
\hline \multirow{2}{*}{ [15] } & [24] & 30.4 & 31.5 & 30.7 & 30.2 & 31.5 & 31.1 \\
\hline & [25] & 30.4 & 31.5 & 31.0 & 30.2 & 31.6 & 31.3 \\
\hline \multicolumn{2}{|c|}{ Joint Demosaicking-Denoising [27] } & 27.1 & 28.6 & 28.3 & 27.1 & 28.8 & 28.5 \\
\hline \multicolumn{2}{|c|}{ Joint Demosaicking-Denoising [30] } & 30.7 & 31.5 & 31.2 & 30.5 & 31.6 & 31.5 \\
\hline \multicolumn{2}{|c|}{ PCA-based CFA Denoising + Demosaicking [4] } & 29.6 & 31.4 & 30.2 & 29.5 & 31.4 & 30.3 \\
\hline \multicolumn{2}{|c|}{ PCA-based CFA Denoising + Demosaicking [9] } & 30.9 & 31.6 & 31.6 & 30.9 & 31.7 & 31.8 \\
\hline \multicolumn{2}{|c|}{ PCA-based CFA Denoising + Demosaicking [10] } & 30.8 & 31.5 & 31.4 & 30.7 & 31.5 & 31.5 \\
\hline \multicolumn{2}{|c|}{ PCA-based CFA Denoising + Demosaicking [15] } & 30.7 & 31.5 & 31.5 & 30.7 & 31.6 & 31.7 \\
\hline
\end{tabular}

TABLE III

PSNR (DB) Results of the ReConstructed Houses ImAGeS by Different Demosaicking AND Denoising Methods

\begin{tabular}{|c|c|c|c|c|c|c|c|}
\hline \multirow{3}{*}{ Demosaicking Methods } & \multirow{3}{*}{ Denoising Methods } & \multicolumn{5}{|c|}{ PSNR Results (dB) } \\
\cline { 3 - 8 } & & $\sigma_{r}=\sigma_{g}=\sigma_{b}=12$ & \multicolumn{2}{|c|}{$\sigma_{r}=13, \sigma_{g}=12, \sigma_{b}=10$} \\
\cline { 2 - 7 } & {$[24]$} & $\mathrm{R}$ & $\mathrm{G}$ & $\mathrm{B}$ & $\mathrm{R}$ & $\mathrm{G}$ & $\mathrm{B}$ \\
\hline \multirow{2}{*}[4]{} & {$[25]$} & 25.9 & 27.9 & 25.9 & 25.8 & 27.9 & 26.2 \\
\cline { 2 - 8 } & {$[24]$} & 28.2 & 29.1 & 28.2 & 28.0 & 29.1 & 28.5 \\
\hline \multirow{2}{*}[9]{} & {$[25]$} & 28.2 & 28.8 & 28.3 & 28.0 & 28.9 & 28.6 \\
\hline \multirow{2}{*}[10]{} & {$[24]$} & 27.4 & 28.3 & 27.5 & 27.4 & 28.4 & 27.7 \\
\hline \multirow{2}{*}[15]{} & {$[25]$} & 27.6 & 28.5 & 27.8 & 27.6 & 28.7 & 28.1 \\
\hline & {$[24]$} & 28.4 & 29.5 & 28.6 & 28.3 & 29.6 & 28.8 \\
\hline \multirow{2}{*}{ Joint Demosaicking-Denoising [27] } & $\mathbf{2 8 . 6}$ & $\mathbf{2 9 . 6}$ & 28.9 & 28.5 & $\mathbf{2 9 . 7}$ & $\mathbf{2 9 . 2}$ \\
\hline Joint Demosaicking-Denoising [30] & 24.7 & 26.3 & 24.9 & 24.7 & 26.3 & 25.0 \\
\hline PCA-based CFA Denoising + Demosaicking [4] & 28.3 & 29.4 & 28.7 & 28.5 & 28.2 & 29.3 & 28.8 \\
\hline PCA-based CFA Denoising + Demosaicking [9] & $\mathbf{2 8 . 6}$ & 29.5 & $\mathbf{2 9 . 0}$ & $\mathbf{2 8 . 6}$ & 29.5 & 29.1 \\
\hline PCA-based CFA Denoising + Demosaicking [10] & 28.2 & 29.1 & 28.6 & 28.2 & 29.2 & 28.7 \\
\hline PCA-based CFA Denoising + Demosaicking [15] & 28.5 & 29.4 & 28.9 & 28.5 & 29.5 & 29.1 \\
\hline
\end{tabular}

in Fig. 5(a) and (b). These test images with resolution of $512 \times 768$ pixels are widely used in the literature for evaluating demosaicking algorithms and among the most difficult ones in the well-known Kodak dataset. Following the standard practice, mosaic CFA images were obtained by sampling the test images using the Bayer pattern. To simulate CFA data with channel-dependent sensor noise, Gaussian white noise was added separately to red, blue and green channels of the mosaic images. For a fair evaluation of the proposed scheme with the other denoising schemes, we suppose these algorithms work on an equivalent channel-independent Gaussian white noise of standard deviation $\sigma_{a}=(1 / 2) \sqrt{\sigma_{r}^{2}+2 \sigma_{g}^{2}+\sigma_{b}^{2}}$, i.e., the average energy of the channel-dependent noises, in the first scenario. Also, we evaluate both stationary and nonstationary noise situations, namely when $v_{r}, v_{g}$ and $v_{b}$ are equal and when they are not equal. In the later case, we make the noise level of each channel proportional to the signal energy of that channel.

Since original CFA images are available, the performance of the different denoising solutions can be objectively evaluated using the peak signal-to-noise ratio (PSNR) of the denoised images with respect to the original images. Table I lists the achieved PSNR values of them on the two test images. We see that the proposed spatially adaptive PCA-based CFA denoising scheme achieves the highest PSNR values and outperforms the other wavelet-based denoising solutions. It is also observed that by dividing the whole big CFA image into four monochromatic sub-images, the denoising performance can be improved for the schemes in [24] and [25]. Fig. 6 illustrates the denoising results of CFA image "fence" by showing cropped difference images between the denoised CFA images and the original CFA image. 


\section{B. Joint Assessment on Denoising and Demosaicking Using Simulated CFA Images}

It is necessary to evaluate the proposed CFA denoising scheme in joint with demosaicking. Since the proposed approach performs denoising before demosaicking, it is compared with two other approaches: denoising after demosaicking and joint denoising-demosaicking, which constitute state-of-the-art schemes to suppress noise in single-sensor camera images. ${ }^{2}$ Namely, eight solutions by combining four powerful demosaicking techniques [4], [9], [10], [15] and two sophisticated wavelet-based denoising techniques [24], [25] to restore the full-color information from CFA data sequentially, and two recently developed joint demosaicking-denoising schemes [27], [30] were employed for comparison. Finally, the competition is completed by four representatives of the denoising before demosaicking approach, obtained by combining the proposed CFA image denoising method and the demosaicking techniques [4], [9], [10], [15].

As in Section IV-A, the example images in Fig. 5(a) and (b) were used in the experiments. The noisy CFA images were input to the competitive solutions to produce final restored images (i.e., demosaicked full-color data with suppressed noise). Tables II and III list the achieved PSNR values by different schemes. Detailed inspection of the results indicates that the proposed method produces very good results and outperforms many prior methods. When working with each one of the four demosaicking algorithms, the proposed PCA-based CFA denoising method almost always gives better result than the wavelet-based denoising schemes with the corresponding demosaicking algorithm. The proposed CFA denoising method followed by demosaicking method [9] performs better than both the two recently proposed joint demosaicking-denoising schemes in [27] and [30].

In addition to the good PSNR results, one important advantage of the proposed method is characterized by high visual quality of the final restored images. To save space while demonstrating the visual differences between the images restored using various solutions, only cropped parts of the final full-color images are shown in the paper. The restored images in the original resolution are available at http://www.comp.polyu.edu.hk/ cslzhang/PCA-CFA-Denoisin g.htm. Since the denoising scheme from [25] gives a little higher visual quality than [24], the outputs of the denoising after demosaicking approach are produced for visual comparisons only with the denoising method from [25] coupled with the powerful demosaicking techniques presented in [9], [10], and [15]. To show the output of the denoising before demosaicking approach, the proposed CFA denoising scheme is coupled with the demosaicking solution of [9] as it gives the best result among the demosaicking schemes [4], [9], [10], [15]. Both the outputs of joint demosaicking-denoising solutions [27], [30] are presented for visual comparisons.

Fig. 7(a) shows parts of the test image fence and Fig. 7(b) is the simulated noisy CFA image. The noise levels are $\sigma_{r}=13$, $\sigma_{g}=12, \sigma_{b}=10$. Fig. 7(c)-(e) show, respectively, the images restored by applying denoising method of [25] to the outputs

${ }^{2}$ We thank the authors of [10], [15], and [27] for sharing their codes.

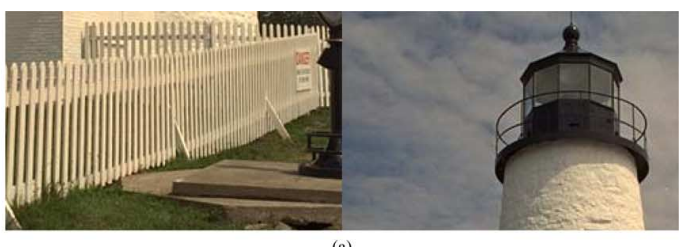

(a)

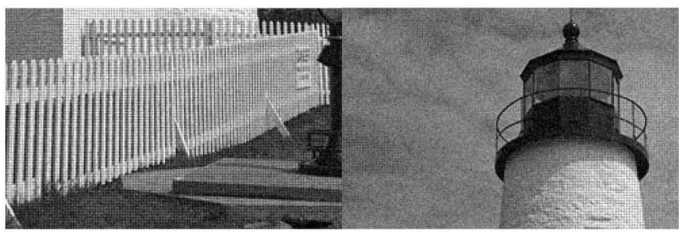

(b)

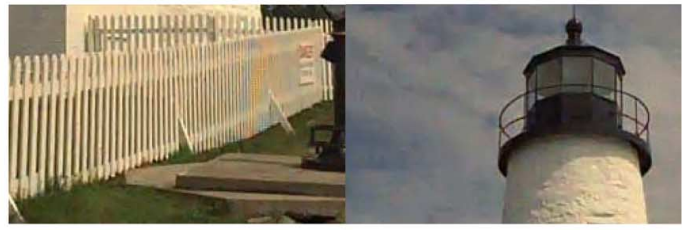

(c)

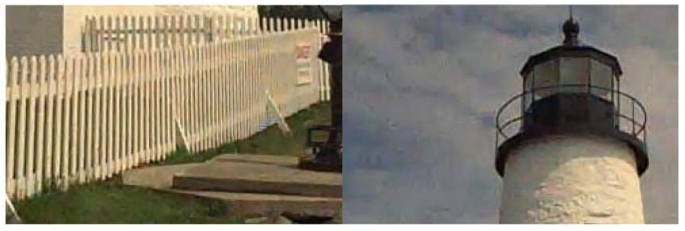

(d)

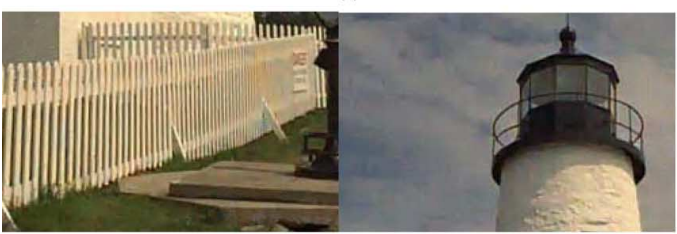

(e)

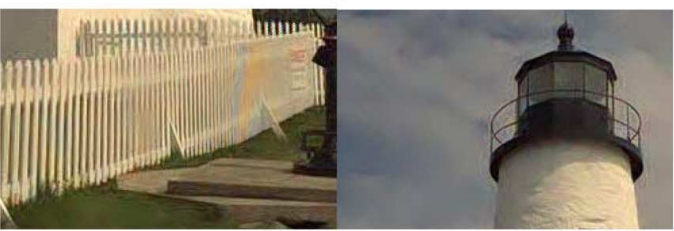

(f)

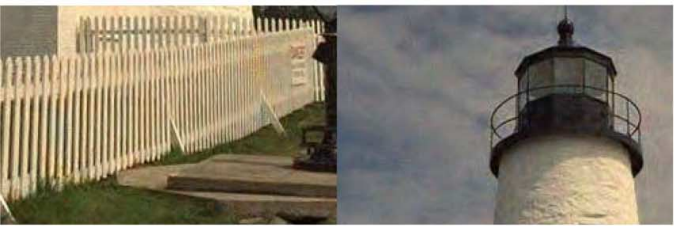

(g)

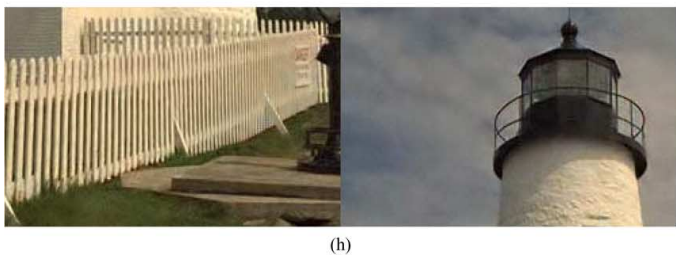

Fig. 7. Cropped images of the reconstructed color "fence" image. (a) Original image; (b) noisy CFA data $\left(\sigma_{r}=13, \sigma_{g}=12, \sigma_{b}=10\right)$; (c)-(e) are reconstructed by demosaicking methods [9], [10] and [15] followed by denoising method [25]; (f) and (g) are reconstructed by joint demosaicking-denoising methods [27] and [30]; (h) is reconstructed by the proposed adaptive PCA-based CFA denoising method followed by demosaicking method [9].

of the demosaicking methods of [9], [10] and [15]. Fig. 7(f) and $(\mathrm{g})$ correspond, respectively, to the output of the joint de- 


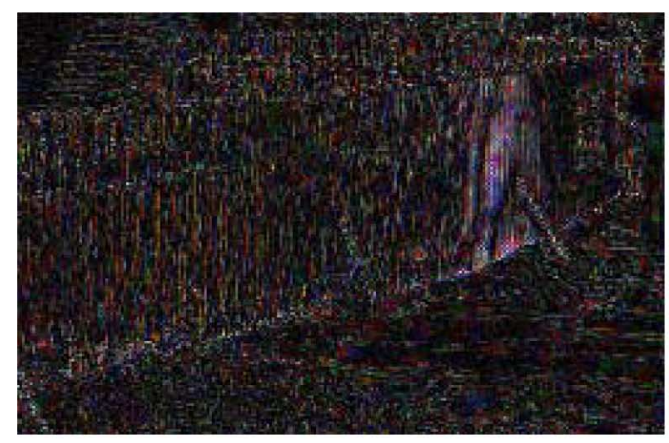

(a)

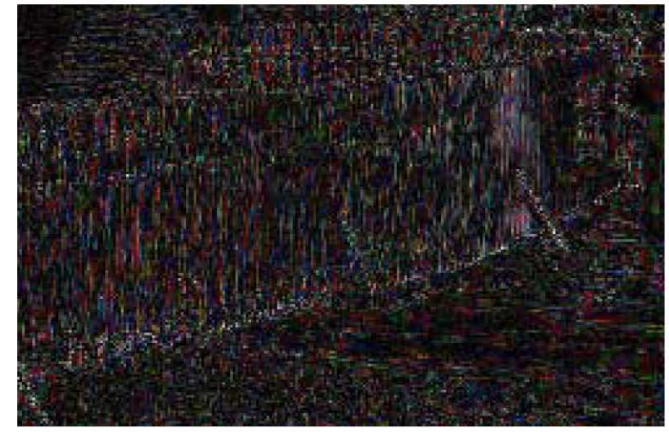

(c)

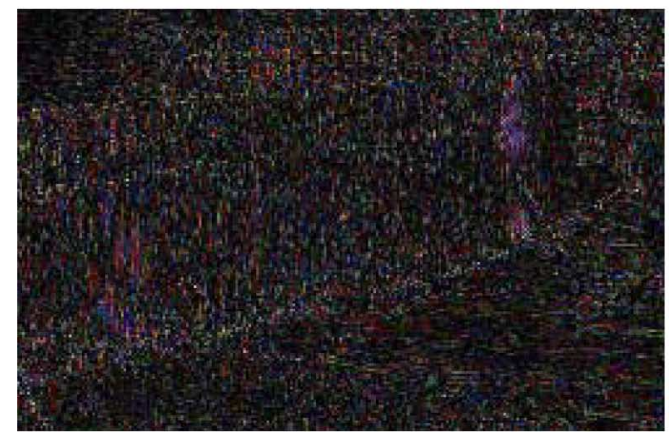

(e)

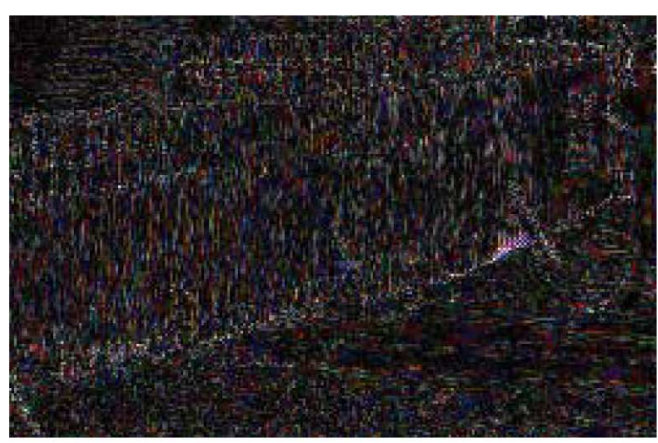

(b)

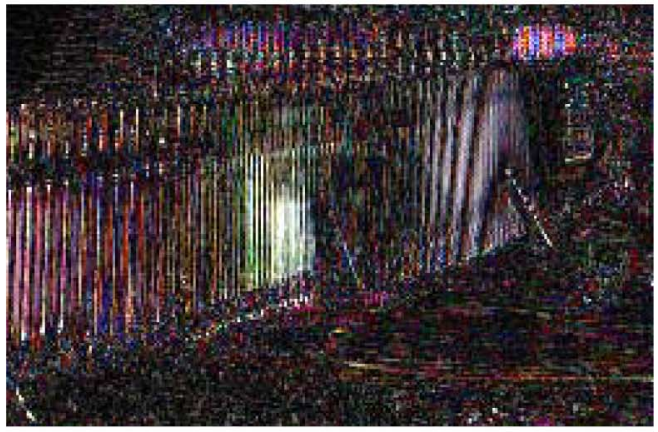

(d)

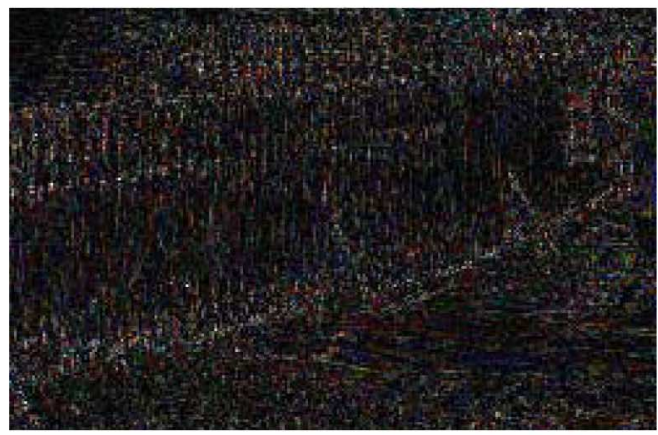

(f)

Fig. 8. (a)-(f) Color difference images between the original image in Fig. 7(a) and the restored images in Fig. 7(c)-(h), respectively.

mosaicking-denoising methods from [27] and [30]. Fig. 7(h) is the reconstructed image by the proposed adaptive PCA-based CFA denoising method followed by demosaicking method [9]. Fig. 8(a)-(f) shows the color difference images between the restored images in Fig. 7(c)-(h) and the original image in Fig. 7(a). Similarly, Fig. 9 shows the results produced by the same set of methods on the houses image.

Detailed inspection of the restored images shown in Figs. 7-9 reveals that even the sophisticated demosaicking methods in [9], [10], and [15] usually generate many noise-caused color artifacts that resist the subsequent denoising process. Moreover, denoising the demosaicked image blurs edges and can remove fine details while suppressing the color artifacts. When restoring the images via joint demosaicking-denoising, the method in [30] performs better than [27], but both the two methods suffer from many color artifacts. Compared with the prior solutions, by performing the denoising operations directly on CFA data the proposed spatially adaptive PCAbased denoising method is more effective in removing noise, thus reducing significantly the amount of the sensor noisecaused color artifacts in the restored images. In addition to the improved noise reduction performance, the proposed approach well preserves the fine image structures and achieves the best visual quality among the tested solutions. This can be clearly seen in the grass portion of the fence image in Figs. 7 and 8, and the window panel portion of the houses image in Fig. 9. Please note that those detailed structures are over-smoothed by other schemes when combating noise, but preserved when using the proposed adaptive PCA analysis-based method.

\section{Experiments on Real CFA Image}

In addition to the test images, the methods under consideration were applied to the real single-sensor captured images. Fig. 5(c) shows an example CFA image "resolution" with size of $500 \times 800$ pixels, cropped from a frame captured by the 


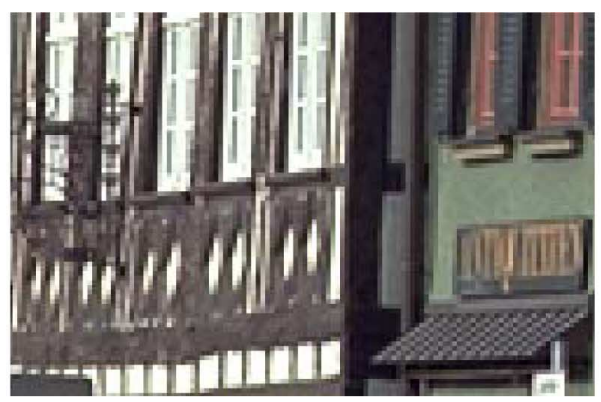

(a)

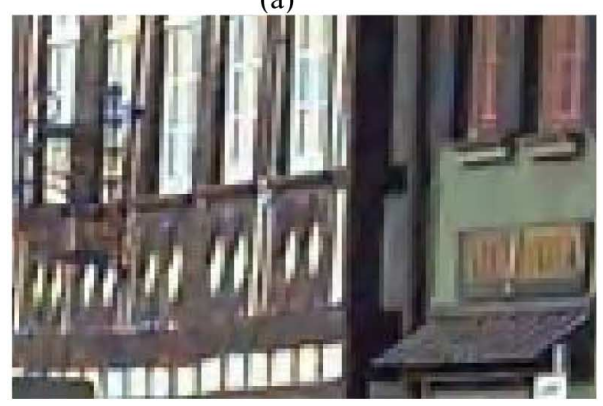

(c)

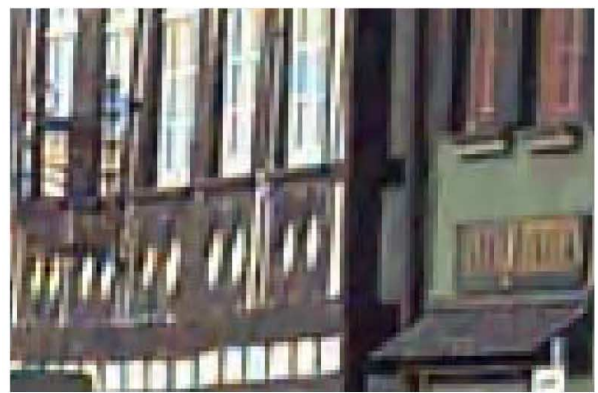

(e)

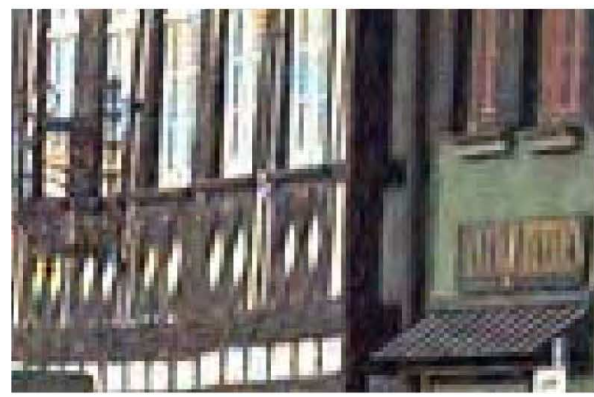

(g)

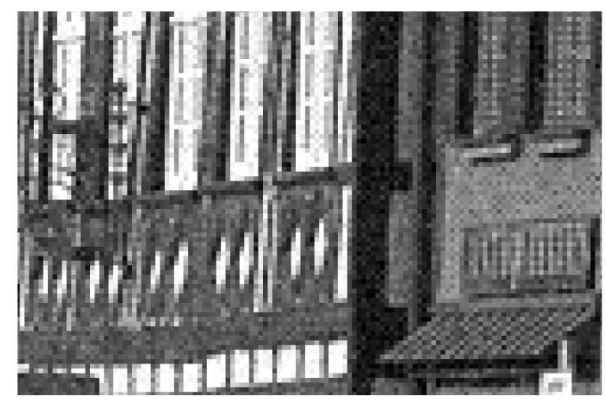

(b)

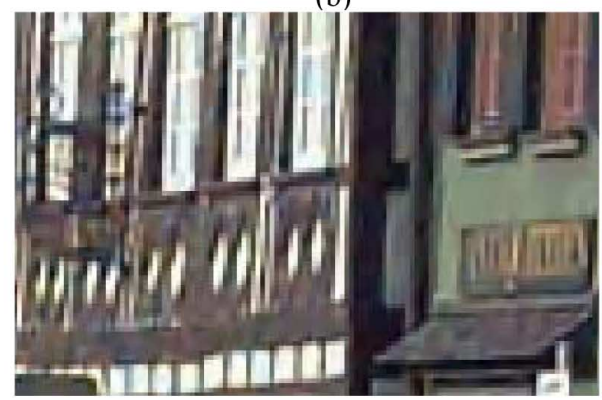

(d)

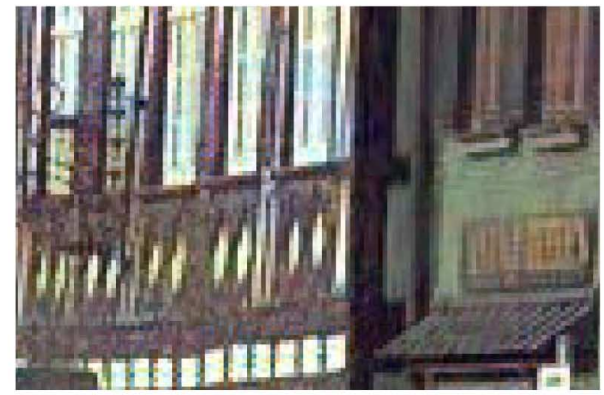

(f)

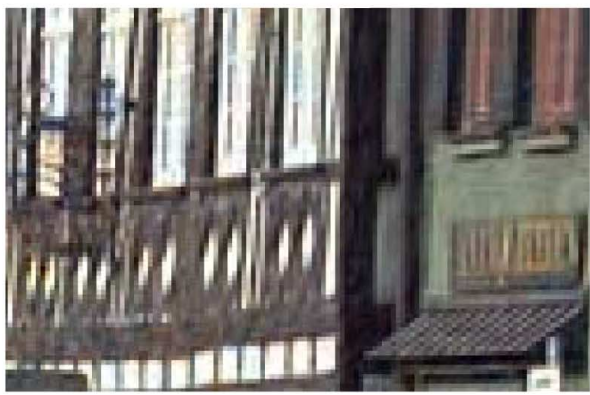

(h)

Fig. 9. Cropped and zoom-in images of the reconstructed color "house" image. (a) Original image; (b) noisy CFA data $\left(\sigma_{r}=13, \sigma_{g}=12, \sigma_{b}=10\right) ;(\mathrm{c})-(\mathrm{e})$ are reconstructed by demosaicking methods [9], [10] and [15] followed by denoising method [25]; (f) and (g) are reconstructed by joint demosaicking-denoising methods [27] and [30]; (h) is reconstructed by the proposed adaptive PCA-based CFA denoising method followed by demosaicking method [9].

digital cinema camera under relatively weak illumination. ${ }^{3}$ Denoising such real CFA images using the proposed method requires calculating the noise energy of each channel from the CFA data. Specifically, we divide the $N \times M$ CFA image into four $N / 2 \times M / 2$ sub-images (two green sub-images, one red sub-image and one blue sub-image). We then apply one-stage orthogonal wavelet transform [18] to each sub-image. The noise level can be estimated as $\sigma=\operatorname{Median}(\mathbf{w}) / 0.6475$ [19]

${ }^{3}$ We thank the DALSA Corporation, Waterloo, Canada, for providing us the test image. or $\sigma=\sqrt{(1 / N \cdot M) \sum_{i=1}^{N} \sum_{j=1}^{M} \mathbf{w}^{2}(i, j)}$, where $\mathbf{w}$ is the diagonal sub-band at the first stage. (The second method is used here.) For the green channel, the noise level is the average of $\sigma$ 's from the two green sub-images. Fig. 10 presents the results in the same experimental setting as in the previous experiments. It is not difficult to see that the proposed method outperforms the competition in terms of visual quality in the realistic imaging scenarios as well. This is due to the fact that the proposed method efficiently uses spatial and spectral image characteristics to suppress noise and preserve edges and fine 

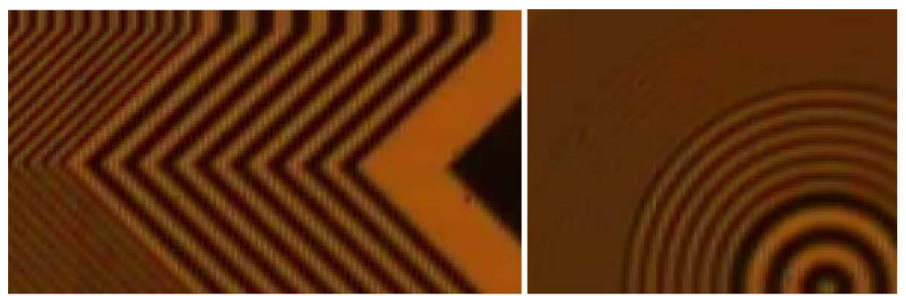

(a)

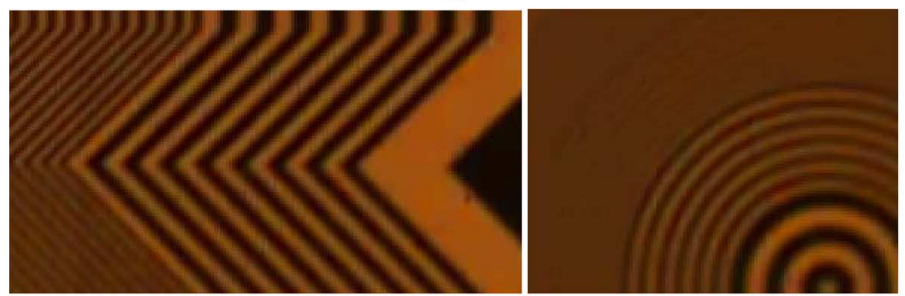

(b)

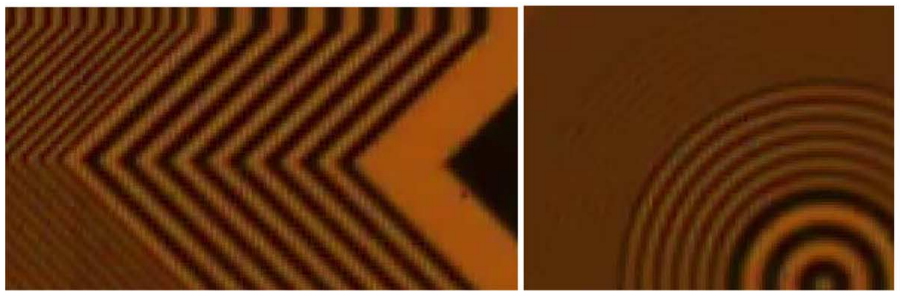

(c)

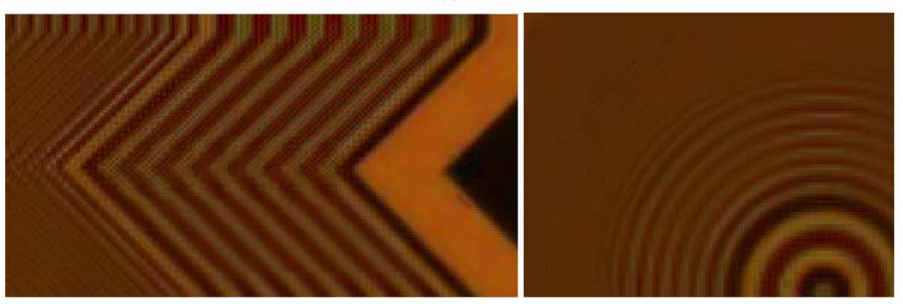

(d)

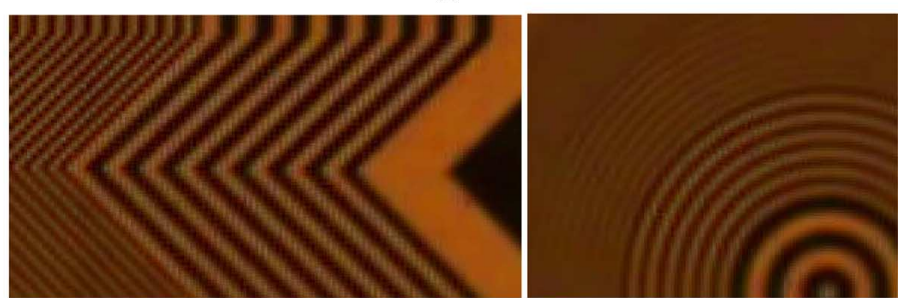

(e)

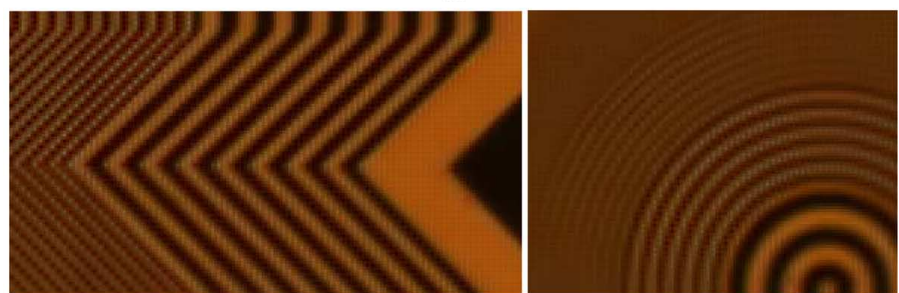

(f)

Fig. 10. Cropped and zoom-in images of the reconstructed color "resolution" image. (a)-(c) are reconstructed by demosaicking methods [9], [10], [15] followed by denoising method [25]; (d) and (e) are reconstructed by joint demosaicking-denoising methods [27], [30]; (f) is reconstructed by the proposed adaptive PCA-based CFA denoising method followed by demosaicking method [9].

details, simultaneously. Many noise-caused color artifacts in the "demosaicking first and denoising later" schemes and the joint demosaicking and denoising schemes are removed in the proposed "denoising first and demosaicking later" scheme, while many edges smoothed in other schemes are preserved in the proposed scheme.

\section{CONCLUSION}

This paper presented a PCA-based CFA image denoising scheme for single-sensor digital camera imaging applications. To fully exploit the spatial and spectral correlations of the CFA sensor readings during the denoising process, the mosaic 
samples from different color channels were localized by using a supporting window to constitute a vector variable, whose statistics are calculated to find the PCA transform matrix. The dimension reduction and LMMSE were conducted in the PCA transformed domain to suppress the CFA image noise. The denoising procedure is completed by inversing the PCA transform to produce the enhanced CFA image. To further reduce the noise residual in smooth areas and phantom artifacts around the boundary between edges and background, the procedures of CFA image decomposition and training sample selection were introduced before PCA transformation. The proposed direct CFA image denoising scheme, followed by a subsequent demosaicking scheme, reduces significantly the noise-caused color artifacts in the demosaicked images. Such artifacts often appear in the output full-color images of many "demosaicking first and denoising later" schemes as well as some joint demosaicking-denoising schemes. While suppressing noise, the proposed scheme preserves very well the fine structures in the image, which are often smoothed by other denoising schemes.

\section{REFERENCES}

[1] "Color Imaging Array," U.S. patent 3971 065, 1975.

[2] P. Longère, X. Zhang, P. B. Delahunt, and D. H. Brainard, "Perceptual assessment of demosaicing algorithm performance," Proc. IEEE, vol. 90, no. 1, pp. 123-132, Jan. 2002.

[3] J. E. Adams, "Intersections between color plane interpolation and other image processing functions in electronic photography," Proc. SPIE, vol. 2416, pp. 144-151, 1995.

[4] J. E. Adams and J. F. Hamilton, Jr., "Adaptive Color Plane Interpolation in Single Color Electronic Camera," U.S. Patent, 5506 619, 1996.

[5] E. Chang, S. Cheung, and D. Y. Pan, "Color filter array recovery using a threshold-based variable number of gradients," Proc. SPIE, vol. 3650, pp. 36-43, 1999.

[6] R. Kimmel, "Demosaicing: Image reconstruction from CCD samples," IEEE Trans. Image Process., vol. 8, no. 9, pp. 1221-1228, Sep. 1999.

[7] B. K. Gunturk, Y. Altunbasak, and R. M. Mersereau, "Color plane interpolation using alternating projections," IEEE Trans. Image Process., vol. 11, no. 9, pp. 997-1013, Sep. 2002.

[8] B. K. Gunturk, J. Glotzbach, Y. Altunbasak, R. W. Schafer, and R. M. Mersereau, "Demosaicking: Color filter array interpolation in singlechip digital cameras," IEEE Signal Process. Mag., vol. 22, no. 1, pp. 44-54, Jan. 2005.

[9] L. Zhang and X. Wu, "Color demosaicking via directional linear minimum mean square-error estimation," IEEE Trans. Image Process., vol. 14, no. 12, pp. 2167-2178, Dec. 2005.

[10] K. Hirakawa and T. W. Parks, "Adaptive homogeneity-directed demosaicing algorithm," IEEE Trans. Image Process., vol. 14, no. 3, pp. 360-369, Mar. 2005.

[11] R. Lukac and K. N. Plataniotis, "Color filter arrays: design and performance analysis," IEEE Trans. Consum. Electron., vol. 51, no. 11, pp. 1260-1267, Nov. 2005.

[12] R. Lukac and K. N. Plataniotis, "A taxonomy of color image filtering and enhancement solutions," in Advances in Imaging and Electron Physics, P. W. Hawkes, Ed. New York: Elsevier/Academic, 2006, vol. 140, pp. 187-264.

[13] R. Lukac, B. Smolka, K. Martin, K. N. Plataniotis, and A. N. Venetsanopoulos, "Vector filtering for color imaging," IEEE Signal Process. Mag., vol. 22, no. 1, pp. 74-86, Jan. 2005.

[14] R. Lukac, K. Martin, and K. N. Plataniotis, "Demosaicked image postprocessing using local color ratios," IEEE Trans. Circuits Syst. Video Technol., vol. 14, no. 6, pp. 914-920, Jun. 2004.

[15] X. Li, "Demosaicing by successive approximation," IEEE Trans. Image Process., vol. 14, no. 3, pp. 370-379, Mar. 2005.

[16] D. Alleysson, S. Susstrunk, and J. Herault, "Linear demosaicing inspired by the human visual system," IEEE Trans. Image Process., vol. 14, no. 4, pp. 439-449, Apr. 2005.
[17] D. D. Muresan and T. W. Parks, "Demosaicing using optimal recovery," IEEE Trans. Image Process., vol. 14, no. 2, pp. 267-278, Feb. 2005.

[18] S. Mallat, A Wavelet Tour of Signal Processing. New York: Academic, 1998.

[19] D. L. Donoho and I. M. Johnstone, "Ideal spatial adaptation via wavelet shrinkage," Biometrika, vol. 81, pp. 425-455, 1994.

[20] R. R. Coifman and D. L. Donoho, "Translation-invariant de-noising," in Wavelet and Statistics, A. Antoniadis and G. Oppenheim, Eds., 1st ed. Berlin, Germany: Springer-Verlag, 1995.

[21] S. G. Chang, B. Yu, and M. Vetterli, "Spatially adaptive wavelet thresholding with context modeling for image denoising," IEEE Trans. Image Process., vol. 9, no. 9, pp. 1522-1531, Sep. 2000.

[22] L. Zhang, P. Bao, and X. Wu, "Multiscale LMMSE-based image denoising with optimal wavelet selection," IEEE Trans. Circuits Syst. Video Technol., vol. 15, no. 4, pp. 469-481, Apr. 2005.

[23] M. K. Mihçak, I. Kozintsev, K. Ramchandran, and P. Moulin, "Lowcomplexity image denoising based on statistical modeling of wavelet coefficients," IEEE Signal Process. Lett., vol. 6, no. 12, pp. 300-303, Dec. 1999.

[24] A. Pizurica and W. Philips, "Estimating the probability of the presence of a signal of interest in multiresolution single- and multiband image denoising," IEEE Trans. Image Process., vol. 15, no. 3, pp. 654-665, Mar. 2006

[25] J. Portilla, V. Strela, M. J. Wainwright, and E. P. Simoncelli, "Image denoising using scale mixtures of gaussians in the wavelet domain," IEEE Trans. Image Process., vol. 12, no. 11, pp. 1338-1351, Nov. 2003.

[26] D. D. Muresan and T. W. Parks, "Adaptive principal components and image denoising," in Proc. Int. Conf. Image Processing, Sep. 14-17, 2003, vol. 1, pp. I101-I104.

[27] K. Hirakawa and T. W. Parks, "Joint demosaicking and denoising," IEEE Trans. Image Process., vol. 15, no. 8, pp. 2146-2157, Aug. 2006.

[28] K. Hirakawa, X.-L. Meng, and P. J. Wolfe, "A framework for waveletbased analysis and processing of color filter array images with applications to denoising and demosaicing," in Proc. ICASSP, Apr. 2007, vol. 1 , pp. I-597-I-600.

[29] K. Hirakawa and X.-L. Meng, "An empirical bayes EM-wavelet unification for simultaneous denoising, interpolation, and/or demosaicing," in Proc. Int. Conf. Image Process., Oct. 2006, pp. $1453-1456$.

[30] L. Zhang, X. Wu, and D. Zhang, "Color reproduction from noisy CFA data of single sensor digital cameras," IEEE Trans. Image Process., vol. 16, no. 9, pp. 2184-2197, Sep. 2007.

[31] D. Paliy, V. Katkovnik, R. Bilcu, S. Alenius, and K. Egiazarian, "Spatially adaptive color filter array interpolation for noiseless and noisy data," Int. J. Imaging Systems and Technology, Special Issue on Applied Color Image Processing, vol. 17, pp. 105-122, 2007.

[32] D. Paliy, M. Trimeche, V. Katkovnik, and S. Alenius, "Demosaicing of noisy data: spatially adaptive approach," Proc. SPIE, vol. 6497, pp. 64970K-64970K, 2007.

[33] H. J. Trussell and R. E. Hartwig, "Mathematics for demosaicking," IEEE Trans. Image Process., vol. 11, no. 4, pp. 485-492, Apr. 2002.

[34] R. Ramanath and W. E. Snyder, "Adaptive demosaicking," J. Electron. Imag., vol. 12, no. 4, pp. 633-642, Oct. 2003.

[35] A. Foi, S. Alenius, V. Katkovnik, and K. Egiazarian, "Noise measurement for raw-data of digital imaging sensors by automatic segmentation of nonuniform targets," IEEE Sensors J., vol. 7, pp. 1456-1461, 2007.

[36] A. Foi, V. Katkovnik, D. Paliy, K. Egiazarian, M. Trimeche, S. Alenius, R. Bilcu, and M. Vehvilainen, "Apparatus, Method, Mobile Station and Computer Program Product for Noise Estimation, Modeling and Filtering of a Digital Image," U.S. Patent Application No. 11/426,128, 2006.

[37] C. C. Koh, J. Mukherjee, and S. K. Mitra, "New efficient methods of image compression in digital cameras with color filter array," IEEE Trans. Consum. Electron., vol. 49, no. 11, pp. 1448-1456, Nov. 2003.

[38] S. Haykin, Neural Networks: A Comprehensive Foundation, 2nd ed. Englewood Cliffs, NJ: Prentice-Hall, 1999.

[39] K. Fukunaga, Introduction to Statistical Pattern Recognition, 2nd ed. New York: Academic, 1991. 


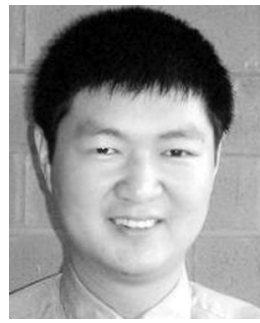

Lei Zhang (M'04) received the B.S. degree in 1995 from the Shenyang Institute of Aeronautical Engineering, Shenyang, China, and the M.S. and $\mathrm{Ph} . \mathrm{D}$. degrees in electrical and engineering from Northwestern Polytechnical University, Xi'an, China, in 1998 and 2001, respectively.

From 2001 to 2002, he was a Research Associate in the Department of Computing, The Hong Kong Polytechnic University. From January 2003 to January 2006, he was a Postdoctoral Fellow in the Department of Electrical and Computer Engineering, McMaster University, Hamilton, ON, Canada. Since January 2006, he has been an Assistant Professor in the Department of Computing, The Hong Kong Polytechnic University. His research interests include image and video processing, biometrics, pattern recognition, multisensor data fusion and optimal estimation theory, etc.

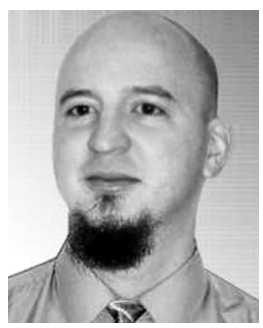

Rastislav Lukac (M'02) received M.S. (Ing.) and $\mathrm{Ph} . \mathrm{D}$. degrees in telecommunications from the Technical University of Kosice, Slovak Republic, in 1998 and 2001, respectively.

From February 2001 to August 2002, he was an Assistant Professor with the Department of Electronics and Multimedia Communications, Technical University of Kosice. From August 2002 to July 2003, he was a researcher with the Slovak Image Processing Center in Dobsina, Slovak Republic. From January 2003 to March 2003, he was a postdoctoral fellow with the Artificial Intelligence and Information Analysis Laboratory, Aristotle University of Thessaloniki, Greece. From May 2003 to August 2006, he was a postdoctoral fellow with the Edward S. Rogers, Sr., Department of Electrical and Computer Engineering, University of Toronto, Toronto, ON, Canada. Since September 2006, he has been a Senior Research Scientist at Epson Edge, Epson Canada, Ltd., Toronto. He is the author of three books and four textbooks, a contributor to eight books, and he has published over 200 scholarly research papers in the areas of digital camera image processing, color image and video processing, multimedia security, and microarray image processing. He is an author of over 20 patent-pending inventions in the areas of digital color imaging and pattern recognition.

Dr. Lukac is a member of the IEEE Circuits and Systems, IEEE Consumer Electronics, and IEEE Signal Processing societies. He is a Digital Imaging and Computer Vision book series editor (CRC Press/Taylor \& Francis). He is an editor of the books Single-Sensor Imaging: Methods and Applications for Digital Cameras (CRC Press/Taylor \& Francis, 2008) and Color Image Processing: Methods and Applications (CRC Press/Taylor \& Francis, 2006). He is a guest editor of the Real-Time Imaging Special Issue on Multi-Dimensional Image Processing, the Computer Vision and Image Understanding Special Issue on Color Image Processing, the International Journal of Imaging Systems and Technology Special Issue on Applied Color Image Processing, and the International Journal of Pattern Recognition and Artificial Intelligence Special Issue on Facial Image Processing and Analysis. He is an associate editor for the Journal of Real-Time Image Processing. He is an editorial board member for the Encyclopedia of Multimedia (Springer, 2008, 2nd Ed.). He is the recipient of the 2003 North Atlantic Treaty Organization/National Sciences and Engineering Research Council of Canada (NATO/NSERC) Science Award and the Most Cited Paper Award for the Journal of Visual Communication and Image Representation for the years 2005-2007.

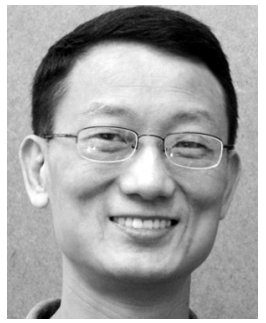

Xiaolin Wu (SM'96) received the B.Sc. degree from Wuhan University, China, in 1982, and the Ph.D. degree from the University of Calgary, Canada, in 1988, both in computer science.

He is currently a Professor in the Department of Electrical and Computer Engineering, McMaster University, Hamilton, ON, Canada, and holds the NSERC-Dalsa Research Chair in digital cinema. His research interests include network-aware multimedia communication, joint source-channel coding, signal quantization and compression, and image processing. He has published over 170 research papers and holds two patents in these fields.

Dr. Wu is an associate editor of the IEEE TRANSACTIONS ON MULTIMEDIA.

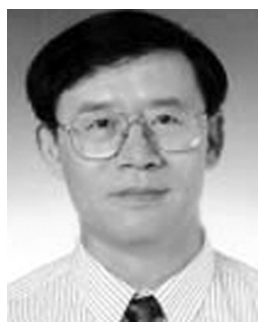

David Zhang (F'08) graduated in computer science from Peking University, China, and received the M.Sc. degree in computer science in 1982 and the Ph.D. degree in 1985 from the Harbin Institute of Technology (HIT), China, and the Ph.D. degree in electrical and computer engineering from the University of Waterloo, Waterloo, ON, Canada, in 1994.

From 1986 to 1988, he was a Postdoctoral Fellow at Tsinghua University, China, and then an Associate Professor at the Academia Sinica, Beijing, China. Currently, he is a Chair Professor at the Hong Kong Polytechnic University where he is the Founding Director of the Biometrics Technology Centre (UGC/CRC) supported by the Hong Kong SAR Government in 1998. He also serves as a Visiting Chair Professor at Tsinghua University an Adjunct Professor at Shanghai Jiao Tong University, Harbin Institute of Technology, and the University of Waterloo. He is the Founder and Editor-in-Chief of the International Journal of Image and Graphics (IJIG); a book editor for the Springer International Series on Biometrics (KISB); an organizer for the International Conference on Biometrics Authentication (ICBA); an associate editor for more than ten international journals; and the author of more than ten books and 200 journal papers

Dr. Zhang is an associate editor for the IEEE TRANSACTIONS ON SYSTEMS, MAN, AND CYBERNETICS A and C. He is also the technical committee Chair of the IEEE CIS. He is a Croucher Senior Research Fellow, Distinguished Speaker of the IEEE Computer Society, and a Fellow of the International Association of Pattern Recognition (IAPR). 